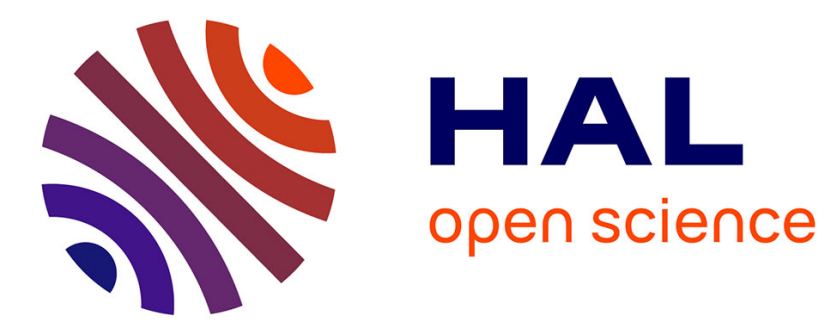

\title{
Some non-stability results for geometric Paneitz-Branson type equations
}

Laurent Bakri, Jean-Baptiste Casteras

\section{To cite this version:}

Laurent Bakri, Jean-Baptiste Casteras. Some non-stability results for geometric Paneitz-Branson type equations. Nonlinearity, 2015, 28 (9), pp.3337-3363. hal-01981194

\section{HAL Id: hal-01981194 \\ https://hal.science/hal-01981194}

Submitted on 14 Jan 2019

HAL is a multi-disciplinary open access archive for the deposit and dissemination of scientific research documents, whether they are published or not. The documents may come from teaching and research institutions in France or abroad, or from public or private research centers.
L'archive ouverte pluridisciplinaire HAL, est destinée au dépôt et à la diffusion de documents scientifiques de niveau recherche, publiés ou non, émanant des établissements d'enseignement et de recherche français ou étrangers, des laboratoires publics ou privés. 


\title{
Some non-stability results for geometric Paneitz-Branson type equations.
}

\author{
Laurent Bakri ${ }^{*} \quad$ Jean-Baptiste Casteras $\ddagger \S$
}

\begin{abstract}
Let $(M, g)$ be a compact riemannian manifold of dimension $n \geq 5$. We consider two Paneitz-Branson type equations with general coefficients

$$
\Delta_{g}^{2} u-\operatorname{div}_{g}\left(A_{g} d u\right)+h u=|u|^{2^{*}-2-\varepsilon} u \text { on } M,
$$

and

$$
\Delta_{g}^{2} u-\operatorname{div}_{g}\left(\left(A_{g}+\varepsilon B_{g}\right) d u\right)+h u=|u|^{2^{*}-2} u \text { on } M,
$$

where $A_{g}$ and $B_{g}$ are smooth symmetric (2,0)-tensors, $h \in C^{\infty}(M)$, $2^{*}=\frac{2 n}{n-4}$ and $\varepsilon$ is a small positive parameter. Under suitable assumptions, we construct solutions $u_{\varepsilon}$ to (??) and (??) which blow up at one point of the manifold when $\varepsilon$ tends to 0 . In particular, we extend the result of Deng and Pistoia 2011 (to the case where $A_{g}$ is the one defined in the Paneitz operator) and the result of Pistoia and Vaira 2013 (to the case $n=8$ and $(M, g)$ locally conformally flat).
\end{abstract}

Keywords: Paneitz-Branson type equations, blow up solutions, LiapunovSchmidt reduction procedure.

Mathematics Subject Classification (2010) : 35J30, 35J60, 35B33, 35 B35.

*Departamento de Matemática, Universidad Técnica Federico Santa María, 1680 Av. españa Valparaíso, Chile. E-mail :laurent.bakri@gmail.com.

${ }^{\dagger}$ The first author was supported by PROYECTO BASAL PFB03 CMM, Universidad de Chile, Santiago (Chile).

${ }_{\ddagger}^{\ddagger}$ UFRGS, Instituto de Matemática, Av. Bento Goncalves 9500, 91540-000 Porto AlegreRS, Brasil Phone : (55) 51 3308-6208. E-mail Jean-Baptiste.Casteras@univ-brest.fr

$\S$ The second author was supported by the CNPq (Brazil) project 501559/2012-4. 


\section{Introduction and statements of the results}

In this paper, we will study the stability of Paneitz type equations in the geometric case for two kinds of perturbations. The Paneitz operator, which is a conformally covariant fourth order operator defined on any pseudoRiemannian manifold, has been introduced by Paneitz in [?]. Branson [?] discovered that this operator describes the conformal transformation of a curvature quantity, the $Q$-curvature. It turns out that this curvature appears in a lot of geometric and physics problems. We refer to the articles of Branson and Gover [?], Chang [?], [?], Chang and Yang [?], and Gursky [?] (and the references therein) for more details on the geometric and physics aspects associated to the notion of $Q$-curvature. Let $(M, g)$ be a compact riemannian manifold of dimension $n \geq 5$. We will be interested in solutions $u \in C^{4, \theta}(M), \theta \in(0,1)$, of the following equation

$$
P_{g} u:=\Delta_{g}^{2} u-\operatorname{div}_{g}\left(A_{g} d u\right)+h u=|u|^{2^{*}-2} u,
$$

where $h \in C^{\infty}(M), 2^{*}=\frac{2 n}{n-4}$ and $A_{g}$ a smooth symmetric $(2,0)$-tensor given by

$$
A_{g}:=\frac{(n-2)^{2}+4}{2(n-1)(n-2)} R_{g} g-\frac{4}{n-2} \operatorname{Ric}_{g}
$$

where $R_{g}$ (resp. $R i c_{g}$ ) stands for the scalar curvature (resp. Ricci curvature) with respect to the metric $g$. When $h$ is given by $h=\frac{n-4}{2} Q_{g}$ where $Q_{g}$ is the $Q$-curvature with respect to the metric $g$ then $P_{g}$ is the so-called PaneitzBranson operator and equation (??) is refered to as the Paneitz-Branson equation. We recall that the $Q$-curvature is defined by

$$
Q_{g}=\frac{1}{2(n-1)} \Delta_{g} R_{g}+\frac{n^{3}-4 n^{2}+16 n-16}{8(n-1)^{2}(n-2)^{2}} R_{g}^{2}-\frac{2}{(n-2)^{2}}\left|R i c_{g}\right|_{g}^{2}
$$

It is well known that the Paneitz operator is conformally invariant, i.e. if $\tilde{g}=\varphi^{\frac{4}{n-4}} g$ then, for all $u \in C^{\infty}(M)$, we have

$$
P_{g}^{n}(u \varphi)=\varphi^{\frac{n+4}{n-4}} P_{\tilde{g}}^{n}(u) .
$$

We point out that if $(M, g)$ is Einstein $\left(\operatorname{Ric}_{g}=\lambda g, \lambda \in \mathbb{R}\right)$, then the PaneitzBranson operator takes the form

$$
P_{g} u=\Delta_{g}^{2} u+b \Delta_{g} u+c u,
$$

where $b=\frac{n^{2}-2 n-4}{2(n-1)} \lambda$ and $c=\frac{n(n-4)\left(n^{2}-4\right)}{16(n-1)^{2}} \lambda$. More generally, following the terminology introduced in [?], when $P_{g}$ is of the form given by (??) 
(respectively by (??)) for arbitrary smooth $(2,0)$ tensor $A_{g}$ and $h \in C^{\infty}(M)$ (respectively for arbitrary real numbers $b$ and $c$ ), the operator $P_{g}$ is referred to as a Paneitz-Branson type operator with general coefficients (respectively Paneitz-Branson type operator with constant coefficients). A lot of attention has been devoted to the study of existence and compactness of solution to (??) (see for example [?, ?, ?, ?, ?, ?] and the references therein). Here, we will be interested in the stability of (??).

In this paper, we will consider two kinds of stability for (??) : the stability with respect to the tensor $A_{g}$ and the stability with respect to the power of the right-side term of (??). More precisely, we say that (??) is exponentstable if, for any sequences of real positive numbers $\left(\varepsilon_{\alpha}\right)_{\alpha}$ such that $\varepsilon_{\alpha} \underset{\alpha \rightarrow \infty}{\longrightarrow} 0$ and for any sequences of solutions $\left(u_{\alpha}\right)_{\alpha} \in C^{4, \theta}(M), \theta \in(0,1)$, of

$$
\Delta_{g}^{2} u_{\alpha}-\operatorname{div}_{g}\left(A_{g} d u_{\alpha}\right)+h u_{\alpha}=\left|u_{\alpha}\right|^{2^{*}-2-\varepsilon_{\alpha}} u_{\alpha},
$$

bounded in $H^{2}(M)$, then up to a subsequence, $u_{\alpha}$ converges in $C^{4}(M)$ to some smooth function $u$ solution of (??). Respectively we say that (??) is $A_{g}$-stable if the functions $u_{\alpha}$ are in fact solutions of

$$
\Delta_{g}^{2} u_{\alpha}-\operatorname{div}_{g}\left(\left(A_{g}+\varepsilon_{\alpha} B\right) d u_{\alpha}\right)+h u_{\alpha}=\left|u_{\alpha}\right|^{2^{*}-2} u_{\alpha},
$$

where $B$ is a smooth symmetric $(2,0)$ tensor. We point out that a related notion of $A_{g}$-stability has been first introduced by Hebey and Robert in [?]. Before, stating more precisely their results, we introduce some notations. We let $\lambda_{i}\left(A_{g}\right)_{x}, x \in M, i=1, \ldots, n$, be the eigenvalues of $A_{g}(x)$ (with respect to the metric $g$ ) repeated with their multiplicity. We define $\lambda_{1}=\inf _{x, i} \lambda_{i}\left(A_{g}\right)_{x}$, $\lambda_{2}=\max _{x, i} \lambda_{i}\left(A_{g}\right)_{x}$ and $S_{w}=\left[\lambda_{1}, \lambda_{2}\right]$. In particular, it is proved in [?] that if $(M, g)$ is locally conformally flat (l.c.f.) and $P_{g}$ is a Paneitz-Branson type operator with strictly positive constant coefficients satisfying $c-\frac{b^{2}}{4}<0$, then (??) is $A_{g}$-stable whenever

a. $b \notin S_{w}$ and $n=6$,

b. $b \neq \frac{\operatorname{Tr} A_{g}}{n}$ if $n \neq 9$ or $n=7$,

c. $b<\frac{\operatorname{Tr} A_{g}}{n}$ if $n=8$.

We point out that the results obtained in [?] are stronger than the ones quoted above. In fact, they show stability of the equation with respect to both $A_{g}$ and $h$. They also obtained the stability when $n=5$ under the hypothesis that the mass of the Green function associated to $P_{g}$ is strictly 
positive. To the authors' knowledge, it is the most refined positive stability result known presently. Concerning non-stability, the first result has been obtained by Deng and Pistoia in [?]. There, they show that, when $A_{g}$ is replaced by some arbitrary smooth $(2,0)$ tensor $B_{g}$, equation (??) is not exponent-stable if

a. $n \geq 7, \operatorname{Tr}_{g}\left(B_{g}-A_{g}\right)$ is not constant and $\min _{M} \operatorname{Tr}_{g}\left(B_{g}-A_{g}\right)>0$,

b. or $n \geq 8$ and $\xi_{0} \in M$ a $C^{1}$ stable critical point of $\operatorname{Tr}_{g}\left(B_{g}-A_{g}\right)$ such that $\operatorname{Tr}_{g}\left(B_{g}-A_{g}\right)\left(\xi_{0}\right)>0$.

A related result has been obtained by the authors in [?] where sign changing blowing-up solutions have been constructed in arbitrary dimensions. We refer to [?] for more details. Recently, Pistoia and Vaira [?] studied the $A_{g^{-}}$ stability of (??) when $P_{g}$ is the Paneitz-Branson operator. They proved that equation (??) is not $A_{g}$-stable, under the following conditions : $(M, g)$ is not conformally flat, $n \geq 9$ and there exists $\xi_{0} \in M$ a $C^{1}$ stable critical point (see below for the definition) of the function $\xi \rightarrow \frac{\operatorname{Tr}_{g} B(\xi)}{\left|W e y l_{g}(\xi)\right|_{g}}$, such that $\operatorname{Tr}_{g} B\left(\xi_{0}\right)>0$. For a function $\phi \in C^{1}(M)$, we recall that a critical point $\xi_{0}$ of $\phi$ is said $C^{1}$ stable if there exists an open neighborhood $\Omega$ of $\xi_{0}$ such that, for any point $\xi \in \bar{\Omega}$, there holds $\nabla_{g} \phi(\xi)=0$ if and only if $\xi=\xi_{0}$ and such that the Brower degree $\operatorname{deg}\left(\nabla_{g} \phi, \Omega, 0\right) \neq 0$. Our first theorem extends [?] to the case where $B_{g}=A_{g}$. More precisely, we have

Theorem 1.1. Let $(M, g)$ be a compact riemannian manifold of dimension $n$, the function $h$ be such that $P_{g}$ is coercive and let $\Phi$ be defined by

$$
\Phi:=-\frac{n^{2}-4 n-4}{96(n-1)(n-3)}\left|W e y l_{g}\right|_{g}^{2}+\frac{1}{n-4}\left(h-\frac{n-4}{2} Q_{g}\right) .
$$

Assume either that :

a. $n \geq 8$ and that $\Phi$ is such that $\min _{x \in M} \Phi(x)>0$.

b. or $n \geq 11$ and there exists $\xi_{0} \in M a C^{1}$ stable critical point of $\Phi$.

Then (??) is not exponent-stable.

As usual for this kind of result, we obtain the previous theorem by constructing a family of solutions $\left(u_{\varepsilon}\right)_{\varepsilon}$ of (??) which blows-up at some point $\xi \in M$ when $\varepsilon$ goes to 0 . More precisely, the family of solutions we construct is of the form

$$
u_{\varepsilon}=\varphi B B l_{\varepsilon}+o(1)
$$


where $o(1) \underset{\varepsilon \rightarrow 0}{\longrightarrow} 0$, and $\varphi$ is a conformal factor, the purpose of which will be precised later (see (??)), and

$$
B B l_{\varepsilon}(x)=\left[n(n-4)\left(n^{2}-4\right)\right]^{\frac{n-4}{8}}\left(\frac{\mu_{\varepsilon}}{\mu_{\varepsilon}+d_{g}\left(x, x_{\varepsilon}\right)^{2}}\right)^{\frac{n-4}{2}},
$$

where $x, x_{\varepsilon} \in M$ and $\mu_{\varepsilon} \in \mathbb{R}^{+}$is such that $\mu_{\varepsilon} \underset{\varepsilon \rightarrow 0}{\longrightarrow} 0$. This form has been introduced by Esposito and Robert in [?]. In our second result we extend the result of [?] to dimension $n=8$ using the same family of blowing-up solutions as the one described above. More precisely, we have

Theorem 1.2. Let $(M, g)$ be a compact riemannian manifold of dimension $n=8$. Assume that $\min _{M}\left\{\left|W e y l_{g}(\xi)\right|_{g}: \operatorname{Tr}_{g}(B)(\xi)>0\right\}>0$. Then (??) is not $A_{g}$-stable.

We would like to make some comments on this theorem. As it was pointed out in [?] (see Remark 3.1), with our approach we are also able to recover the case $n>8$. The approach used in [?] consisted in taking $\varphi \equiv 1$ but adding an higher-order term to the standard bubble $B B l_{\varepsilon}$. The method we use here is more simple but on the other hand, it seems more rigid. Finally, in our last result, we investigated the $A_{g}$-stability when $(M, g)$ is l.c.f. Before stating more precisely our theorem, we introduce some notations. We let $i_{g}$ be the injectivity radius of $(M, g)$ and $r_{0} \in \mathbb{R}_{+}^{*}$ such that $r_{0}<i_{g}$. Since we are assuming that $(M, g)$ is l.c.f., there exists a family $\left(g_{\xi}\right)_{\xi \in M}$ of smooth conformal metrics to $g$ such that $g_{\xi}$ is flat in the geodesic ball $B_{\xi}\left(r_{0}\right)$. We let $G_{g}$ be the Green's function of the Paneitz operator $P_{g}$. We will assume that $G_{g}$ is of the form

$$
G_{g_{\xi}}\left(\exp _{\xi} y, \xi\right)=\frac{1}{\beta_{n}|y|^{n-4}}+A_{\xi}+0^{(4)}(|y|)
$$

where $\beta_{n}=(n-2)(n-4) \omega_{n-1}, \omega_{n-1}=\left|S^{n-1}\right|, A_{\xi}>0$ depending only $(M, g)$ and on $\xi$ (being smooth with respect to $\xi$ ) and $f=O^{(k)}\left(r^{m}\right)$ denotes any quantity satisfying

$$
\left|\nabla^{j} f\left(\exp _{\xi} y\right)\right| \leq C_{j}|y|^{m-j},
$$

for $1 \leq j \leq k$. We have :

Theorem 1.3. Let $(M, g)$ be a locally conformally flat manifold of dimension $n \geq 6$. Assume that $h=Q_{g}$, (??) holds and that $\max _{M} \operatorname{Tr}_{g}(B)>0$. Then (??) is not $A_{g}$-stable. More precisely, for $\varepsilon>0$, there exists a family of solutions $u_{\varepsilon}$ of (??) which blows-up, when $\varepsilon \rightarrow 0$, at some point $\xi_{0}$ so that 
$E\left(\xi_{0}\right)=\max _{M} E(\xi)$ where $E(\xi)=\frac{h(\xi)}{A_{\xi}^{\frac{2}{n-4}}}$. Moreover if $n \geq 7$, for any isolated critical point $\xi_{0}$ of $E$ with non-trivial degree and $\operatorname{Tr}_{g}(B)\left(\xi_{0}\right)>0$, for $\varepsilon>0$, there exists a family of solutions $u_{\varepsilon}$ of (??) which blows-up, when $\varepsilon \rightarrow 0$, $a t \xi_{0}$.

The method used in order to prove the previous theorem is inspired by the one of Esposito, Pistoia and Vétois [?] where a similar result has been proved for the Yamabe equation. The main idea consists in modifying slightly the shape of the family $\left(u_{\varepsilon}\right)_{\varepsilon}$ of blowing-up solutions we are looking for by multiplying the standard bubble $B B l_{\varepsilon}$ by a function depending on the Green function. Finally, we point out that the assumption (??) is very natural. Gursky and Malchiodi in [?] (see Theorem 2.9) proved that if $Q_{g}$ is semi positive, $R_{g} \geq 0,(M, g)$ locally conformally flat but not conformally equivalent to the round sphere, then (??) holds (see also some recent preprints of Hang and Yang for improved results [?]).

The proof of the theorems relies on a well known Lyapunov-Schmidt reduction procedure which permits to reduce the problem to a finite dimensional one for which we defined a reduced energy. The solutions to (??) will then be obtained as critical points of this reduced energy. We refer to [?] and the references therein for more information on the Lyapunov-Schmidt reduction procedure.

The plan of this paper is the following : in section 2, we give some preliminaries. Section 3 is devoted to the proof of Theorems ?? and ?? where the proofs of these two theorems are done in parallel. We begin by giving an estimate of the error and then give an estimate of the reduced energy. Finally, in Section 4, we prove Theorem ??.

\section{Preliminaries.}

Let $\left(\xi_{\alpha}\right)_{\alpha}$ be a sequence of points of $M$. In all the following, we will suppose up to extracting a subsequence that, for $\alpha$ large enough, all the points $\xi_{\alpha}$ belong to a small open set $\Omega$ of $M$ in which there exists a smooth orthogonal frame. Thus, we will identify the tangent spaces $T_{\xi} M$ with $\mathbb{R}^{n}$ for all $\xi \in \Omega$. We recall that we suppose that $P_{g}$ is coercive.

In all the following, we will denote by $\langle., .\rangle_{P_{g}}$, the scalar product, for $u, v \in H^{2}(M)$,

$$
\langle u, v\rangle_{P_{g}}=\int_{M} \Delta_{g} u \Delta_{g} v d V+\int_{M} A_{g}\left(\nabla_{g} u, \nabla_{g} v\right) d V+\int_{M} h u v d V
$$


where here and in the following $d V$ stands for the volume element with respect to the metric $g$. We will denote $\|\cdot\|_{P_{g}}$ the associated norm which is equivalent to the standard norm of $H^{2}(M)$. We denote by $i^{*}: L^{\frac{2 n}{n+4}}(M) \rightarrow$ $H^{2}(M)$ the adjoint operator of the embedding $i: H^{2}(M) \rightarrow L^{\frac{2 n}{n-4}}(M)$, i.e. for all $w \in L^{\frac{2 n}{n+4}}(M)$, the function $u=i^{*}(w) \in H^{2}(M)$ is the unique solution of $\Delta_{g}^{2} u-d i v_{g}\left(A_{g} d u\right)+h u=w$. Using this notation, we see that equations (??) and (??) can be rewritten as, for $u \in H^{2}(M)$,

$$
u=i^{*}\left(f_{\varepsilon}(u)\right),
$$

where $f_{\varepsilon}(u)=|u|^{2^{*}-2-\varepsilon} u$ for (??) and $f_{\varepsilon}(u)=|u|^{2^{*}-2} u-\varepsilon \operatorname{div}_{g}(B(\nabla u)$ for (??). Before proceeding we recall some basic facts. It is well known (see [?]) that all solutions $u \in H^{2}\left(\mathbb{R}^{n}\right)$ of the equation

$$
\Delta_{\text {eucl }}^{2} u=u^{2^{*}-1}=u^{\frac{n+4}{n-4}} \text { in } \mathbb{R}^{n}
$$

are given by

$$
U_{\delta, y}(x)=\delta^{\frac{4-n}{2}} U\left(\frac{x-y}{\delta}\right), \delta>0, y \in \mathbb{R}^{n}
$$

where

$$
U(x)=\left[n(n-4)\left(n^{2}-4\right)\right]^{\frac{n-4}{8}}\left(\frac{1}{1+|x|^{2}}\right)^{\frac{n-4}{2}}=\alpha_{n}\left(\frac{1}{1+|x|^{2}}\right)^{\frac{n-4}{2}} .
$$

It is also well known (see [?]) that all solutions $v \in H^{2}\left(\mathbb{R}^{n}\right)$ of

$$
\Delta_{\text {eucl }}^{2} v=\left(2^{*}-1\right) U^{2^{*}-2} v
$$

are linear combinations of

$$
V_{0}(x)=\alpha_{n} \frac{n-4}{2} \frac{|x|^{2}-1}{\left(1+|x|^{2}\right)^{\frac{n-2}{2}}}
$$

and

$$
V_{i}(x)=\alpha_{n}(n-4) \frac{x_{i}}{\left(1+|x|^{2}\right)^{\frac{n-2}{2}}}, i=1, \ldots, n .
$$

Let us fix $N>n$ and $\xi \in M$, it is well known that there exists $\tilde{g}=\varphi^{\frac{4}{n-2}} g$, $\varphi>0$ is a smooth function on $M$, such that

$$
\operatorname{Ric}_{\tilde{g}}(\xi)=0, \nabla R_{\tilde{g}}(\xi)=0, \Delta_{\tilde{g}} R_{\tilde{g}}(\xi)=\frac{1}{6}\left|W e y l_{g}(\xi)\right|_{g}^{2}
$$

and

$$
d V_{\tilde{g}}=\left(1+O\left(r^{N}\right)\right) d V_{\mathbb{R}^{n}} .
$$


We notice that, in this system of coordinates, we have

$$
Q_{g}(\xi)=\frac{1}{2(n-1)} \Delta_{g} R_{g}(\xi)=\frac{1}{12(n-1)}\left|W e y l_{g}(\xi)\right|_{g}^{2}
$$

We also give the expression of the Paneitz operator for radial function in the previous metric which will be useful in the sequel :

Lemma 2.1. [?, lemma 2.6 p.13] If $u$ is a radial function, then we have

$$
\begin{aligned}
P_{\tilde{g}}(u)=\Delta^{2} u & +\frac{1}{n-2} \nabla_{l} \nabla_{k} R_{g}(0) x^{k} x^{l} A(u)+\frac{n-4}{24(n-1)}\left|W e y l_{g}\right|_{g}^{2}(0) u \\
+ & O\left(r^{3}\right)\left|u^{\prime \prime}\right|+O\left(r^{2}\right)\left|u^{\prime}\right|+O(r) u+O\left(r^{N-1}\right) u^{\prime \prime \prime} \\
& +O\left(r^{N-2}\right) u^{\prime \prime}+O\left(r^{N-3}\right) u^{\prime}
\end{aligned}
$$

where $u^{\prime}=\frac{\partial u}{\partial r}$ and

$$
A(u)=\frac{u^{\prime}}{r}\left(\frac{2(n-1)}{n-2}-\frac{(n-1)(n-2)}{2}+6-n\right)-u^{\prime \prime}\left(\frac{n-2}{2}+\frac{2}{n-2}\right) .
$$

\section{Proofs of Theorems ?? and ??.}

This section will be devoted to the proof of Theorems ?? and ??. Since the form of the solution we construct will be the same in both cases, we will prove Theorems ?? and ?? in parallel. We define, for any real $\delta$ strictly positive, $\xi \in M$ and $x \in M$,

$$
W_{\delta, \xi}(x)=\chi\left(d_{g}(x, \xi)\right) \varphi(x) \delta^{\frac{4-n}{2}} U\left(\delta^{-1} \exp _{\xi}^{-1}(x)\right),
$$

where $d_{g}(x, \xi)$ stands for the distance from $x$ to $\xi$ with respect to the metric $g$, $\exp _{\xi}$ is the exponential map with respect to the metric $g$ and $\chi: \mathbb{R} \rightarrow \mathbb{R}$ is a smooth cutoff function such that $0 \leq \chi \leq 1, \chi(x)=1$ if $x \in\left[-\frac{r_{0}}{2}, \frac{r_{0}}{2}\right]$ and $\chi(x)=0$ if $x \in \mathbb{R} \backslash\left(-r_{0}, r_{0}\right)$. We also define, for any real $\delta$ strictly positive, $\xi \in M$ and $x \in M$,

$$
Z_{\delta, \xi}(x)=\chi\left(d_{g}(x, \xi)\right) \delta^{\frac{n-4}{2}} \frac{d_{g}(x, \xi)^{2}-\delta^{2}}{\left(\delta^{2}+d_{g}(x, \xi)^{2}\right)^{\frac{n-2}{2}}},
$$

and, for $\omega \in T_{\xi} M$,

$$
Z_{\delta, \xi, \omega}(x)=\chi\left(d_{g}(x, \xi)\right) \delta^{\frac{n-2}{2}} \frac{\left\langle\exp _{\xi}^{-1} x, \omega\right\rangle_{g}}{\left(\delta^{2}+d_{g}(x, \xi)^{2}\right)^{\frac{n-2}{2}}} .
$$


We denote by $\Pi_{\delta, \xi}$ respectively $\Pi_{\delta, \xi}^{\perp}$ the projection of $H^{2}(M)$ onto

$$
K_{\delta, \xi}=\operatorname{span}\left\{Z_{\delta, \xi},\left(Z_{\delta, \xi, e_{i}}\right)_{i=1 . . n}\right\}
$$

respectively

$$
K_{\delta, \xi}^{\perp}=\left\{\phi \in H^{2}(M) /\left\langle\phi, Z_{\delta, \xi}\right\rangle_{P_{g}}=0 \text { and }\left\langle\phi, Z_{\delta, \xi, \omega}\right\rangle_{P_{g}}=0, \forall \omega \in T_{\xi} M\right\} .
$$

We are looking for solution $u$ to (??) of the form

$$
u=W_{\delta_{\varepsilon}\left(t_{\varepsilon}\right), \xi_{\varepsilon}}+\phi_{\delta_{\varepsilon}\left(t_{\varepsilon}\right), \xi_{\varepsilon}},
$$

where $\phi_{\delta_{\varepsilon}\left(t_{\varepsilon}\right), \xi_{\varepsilon}} \in K_{\delta_{\varepsilon}\left(t_{\varepsilon}\right), \xi_{\varepsilon}}^{\perp}$ and $\delta_{\varepsilon}\left(t_{\varepsilon}\right) \in \mathbb{R}^{+}$is defined below. It is easy to see that equations (??) (respectively (??)) are equivalent to the following system

$$
\Pi_{\delta_{\varepsilon}(t), \xi}\left(W_{\delta_{\varepsilon}(t), \xi}+\phi_{\delta_{\varepsilon}(t), \xi}-i^{*}\left(f_{\varepsilon}\left(W_{\delta_{\varepsilon}(t), \xi}+\phi_{\delta_{\varepsilon}(t), \xi}\right)\right)\right)=0,
$$

and

$$
\Pi_{\delta_{\varepsilon}(t), \xi}^{\perp}\left(W_{\delta_{\varepsilon}(t), \xi}+\phi_{\delta_{\varepsilon}(t), \xi}-i^{*}\left(f_{\varepsilon}\left(W_{\delta_{\varepsilon}(t), \xi}+\phi_{\delta_{\varepsilon}(t), \xi}\right)\right)\right)=0,
$$

where $f_{\varepsilon}$ is defined by $f_{\varepsilon}(u)=|u|^{2^{*}-2-\varepsilon} u$ (respectively by $f_{\varepsilon}(u)=|u|^{2^{*}-2} u-$ $\left.\varepsilon \operatorname{div}_{g}(B(\nabla u))\right)$. We define $\delta_{\varepsilon}\left(t_{\varepsilon}\right)$, for $t_{\varepsilon}>0$ by

$$
\delta_{\varepsilon}\left(t_{\varepsilon}\right)= \begin{cases}\left(t_{\varepsilon} \varepsilon\right)^{\frac{1}{4}}, & \text { if } n \geq 9, \\ t_{\varepsilon} l^{-1}(\varepsilon), & \text { if } n=8\end{cases}
$$

where $l:\left(0, e^{-\frac{1}{2}}\right) \rightarrow\left(0, e^{-\frac{1}{2}}\right)$ is defined by $l(\delta)=-\delta^{4} \ln \delta$ if $f_{\varepsilon}(u)=|u|^{2^{*}-2-\varepsilon} u$ and by $l(\delta)=-\delta^{2} \ln \delta$ if $f_{\varepsilon}(u)=|u|^{2^{*}-2} u-\varepsilon \operatorname{div}_{g}(B(\nabla u))$.

Let us now give the plan of this section. We will begin by solving (??) in Section 3.1. In Section 3.2, we will solve (??) by proving an estimate of the reduced energy (see Propositions ?? and ??) and give the proof of the theorems. Finally, in Section 3.3, we finish the proof of Proposition ?? by showing that the estimate of the reduced energy holds $C^{1}$-uniformly when $n \geq 11$.

\subsection{Finite dimensional reduction.}

We begin by solving (??). The following proposition is well known and we refer to [?] and [?] for a proof of it. 
Proposition 3.1. Given two real numbers $a<b$, there exists a positive constant $C_{a, b}$ such that for $\varepsilon$ small, for any $t \in[a, b]$ and any $\xi \in M$, there exists a unique function $\phi_{\delta_{\varepsilon}(t), \xi} \in K_{\delta_{\varepsilon}(t), \xi}^{\perp}$ which solves equation (??) and satisfies

$$
\left\|\phi_{\delta_{\varepsilon}(t), \xi}\right\|_{P_{g}} \leq C_{a, b}\left\|i^{*}\left(f_{\varepsilon}\left(W_{\delta_{\varepsilon}(t), \xi}\right)\right)-W_{\delta_{\varepsilon}(t), \xi}\right\|_{P_{g}} .
$$

Moreover, $\phi_{\delta_{\varepsilon}(t), \xi}$ is continuously differentiable with respect to $t$ and $\xi$.

The next two lemma are devoted to estimate $\left\|i^{*}\left(f_{\varepsilon}\left(W_{\delta_{\varepsilon}(t), \xi}\right)\right)-W_{\delta_{\varepsilon}(t), \xi}\right\|_{P_{g}}$ in term of $\varepsilon$. We begin by the case $f_{\varepsilon}(u)=|u|^{2^{*}-2-\varepsilon} u$ (i.e. by (??)).

Lemma 3.2. Assume that $n \geq 8$ and $f_{\varepsilon}(u)=|u|^{2^{*}-2-\varepsilon} u$. Given two positive real numbers $a<b$, there exists a positive constant $C_{a, b}^{\prime}$ such that for $\varepsilon$ small, for any real number $t \in[a, b]$ and any point $\xi \in M$, there holds

$$
\begin{aligned}
& \| i^{*}\left(f_{\varepsilon}\left(W_{\delta_{\varepsilon}(t), \xi}\right)\right)-W_{\delta_{\varepsilon}(t), \xi} \|_{P_{g}} \\
& \leq C_{a, b}^{\prime}\left(\varepsilon\left|\ln \delta_{\varepsilon}(t)\right|+\left\{\begin{array}{ll}
\delta_{\varepsilon}(t)^{\frac{n-4}{2}}, & \text { if } n<12, \\
\delta_{\varepsilon}(t)^{4}\left|\ln \delta_{\varepsilon}(t)\right|, & \text { if } n \geq 12
\end{array}\right) .\right.
\end{aligned}
$$

Proof. All the estimates will be uniform in $t, \xi$ and $\varepsilon$. Since $i^{*}$ is continuous, we have

$$
\begin{aligned}
\| i^{*}\left(f_{\varepsilon}\left(W_{\delta_{\varepsilon}(t), \xi}\right)\right)- & W_{\delta_{\varepsilon}(t), \xi} \|_{P_{g}} \\
& =O\left(\left\|\left(f_{\varepsilon}\left(W_{\delta_{\varepsilon}(t), \xi}\right)\right)-P_{g}\left(W_{\delta_{\varepsilon}(t), \xi}\right)\right\|_{L^{\frac{2 n}{n+4}}}\right) .
\end{aligned}
$$

The triangular inequality yields to

$$
\begin{aligned}
\| i^{*}\left(f_{\varepsilon}\left(W_{\delta_{\varepsilon}(t), \xi}\right)\right. & -W_{\delta_{\varepsilon}(t), \xi} \|_{P_{g}} \\
\leq & C\left\|f_{\varepsilon}\left(W_{\delta_{\varepsilon}(t), \xi}\right)-f\left(W_{\delta_{\varepsilon}(t), \xi}\right)\right\|_{L^{\frac{2 n}{n+4}}} \\
& +C\left\|f\left(W_{\delta_{\varepsilon}(t), \xi}\right)-P_{g}\left(W_{\delta_{\varepsilon}(t), \xi}\right)\right\|_{L^{\frac{2 n}{n+4}}} \\
\leq & C\left(I_{1}+I_{2}\right) .
\end{aligned}
$$

It is easy to see (see for instance inequality (3.28) of [?]) that

$$
I_{1}=\left\|f_{\varepsilon}\left(W_{\delta_{\varepsilon}(t), \xi}\right)-f_{0}\left(W_{\delta_{\varepsilon}(t), \xi}\right)\right\|_{L^{\frac{2 n}{n+4}}}=O\left(\varepsilon\left|\ln \delta_{\varepsilon}(t)\right|\right) .
$$

Using that the Paneitz operator P Paneitz, (i.e. for which $h=\frac{n-4}{2}$ ) is a conformal operator and denoting $\tilde{W}_{\delta_{\varepsilon}(t), \xi}=\frac{W_{\delta_{\varepsilon}(t), \xi}}{\varphi}$, we have

$$
f\left(W_{\delta_{\varepsilon}(t), \xi}\right)-P_{g}^{\text {paneitz }}\left(W_{\delta_{\varepsilon}(t), \xi}\right)=\varphi^{2^{*}-1}\left(f\left(\tilde{W}_{\delta_{\varepsilon}(t), \xi}\right)-P_{\tilde{g}}^{\text {paneitz }}\left(\tilde{W}_{\delta_{\varepsilon}(t), \xi}\right)\right),
$$


where $\tilde{g}$ is the metric defined in (??). Now, since $\tilde{W}_{\delta_{\varepsilon}(t), \xi}$ is a radial function, using Lemma ??, we have

$$
\begin{aligned}
f\left(W_{\delta_{\varepsilon}(t), \xi}\right)-P_{g}\left(W_{\delta_{\varepsilon}(t), \xi}\right)= & f\left(W_{\delta_{\varepsilon}(t), \xi}\right)-P_{g}^{\text {paneitz }}\left(W_{\delta_{\varepsilon}(t), \xi}\right) \\
& +\left(\frac{n-4}{2} Q-h\right) W_{\delta_{\varepsilon}(t), \xi} \\
= & \varphi^{2^{*}-1}\left(f\left(\tilde{W}_{\delta_{\varepsilon}(t), \xi}\right)-\Delta_{\mathbb{R}^{n}}^{2}\left(\tilde{W}_{\delta_{\varepsilon}(t), \xi}\right)\right) \\
& +O\left(r^{2} \partial_{r r} \tilde{W}_{\delta_{\varepsilon}(t), \xi}+r \partial_{r} \tilde{W}_{\delta_{\varepsilon}(t), \xi}+\tilde{W}_{\delta_{\varepsilon}(t), \xi}\right) \\
= & O\left(r^{2} \partial_{r r} \tilde{W}_{\delta_{\varepsilon}(t), \xi}+r \partial_{r} \tilde{W}_{\delta_{\varepsilon}(t), \xi}+\tilde{W}_{\delta_{\varepsilon}(t), \xi}\right) .
\end{aligned}
$$

Direct computations give

$$
\begin{aligned}
\max \left\{\left\|\tilde{W}_{\delta_{\varepsilon}(t), \xi}\right\|_{L^{\frac{2 n}{n+4}}},\right. & \left.\left\|r \partial_{r} \tilde{W}_{\delta_{\varepsilon}(t), \xi}\right\|_{L^{\frac{2 n}{n+4}}},\left\|r^{2} \partial_{r r} \tilde{W}_{\delta_{\varepsilon}(t), \xi}\right\|_{L^{\frac{2 n}{n+4}}}\right\} \\
& \leq C \begin{cases}\delta_{\varepsilon}(t)^{4}, & \text { if } n \geq 13, \\
\delta_{\varepsilon}(t)^{4}\left|\ln \delta_{\varepsilon}(t)\right|, & \text { if } n=12, \\
\delta_{\varepsilon}(t)^{\frac{n-4}{2}}, & \text { if } 5 \leq n \leq 11 .\end{cases}
\end{aligned}
$$

Therefore, we deduce that

$$
\left|I_{2}\right| \leq C \begin{cases}\delta_{\varepsilon}(t)^{4}, & \text { if } n \geq 13 \\ \delta_{\varepsilon}(t)^{4}\left|\ln \delta_{\varepsilon}(t)\right|, & \text { if } n=12 \\ \delta_{\varepsilon}(t)^{\frac{n-4}{2}}, & \text { if } 5 \leq n \leq 11\end{cases}
$$

Combining (??), (??) and (??), the proof of the lemma follows.

Next we prove the equivalent of the previous lemma for equation (??).

Lemma 3.3. Assume that $n=8$ and $f_{\varepsilon}(u)=|u|^{2^{*}-2} u-\varepsilon \operatorname{div}_{g}(B(\nabla u))$. Given two positive real numbers $a<b$, there exists a positive constant $C_{a, b}^{\prime}$ such that for $\varepsilon$ small, for any real number $t \in[a, b]$ and any point $\xi \in M$, there holds

$$
\left\|i^{*}\left(f_{\varepsilon}\left(W_{\delta_{\varepsilon}(t), \xi}\right)\right)-W_{\delta_{\varepsilon}(t), \xi}\right\|_{P_{g}} \leq C_{a, b}^{\prime}\left(\delta_{\varepsilon}(t)^{2}+\varepsilon \delta_{\varepsilon}(t)^{2}\left|\ln \delta_{\varepsilon}(t)\right|^{\frac{3}{4}}\right) .
$$

Proof. Using the continuity of $i^{*}$ and the triangular inequality, we have

$$
\begin{aligned}
& \left\|i^{*}\left(f_{\varepsilon}\left(W_{\delta_{\varepsilon}(t), \xi}\right)\right)-W_{\delta_{\varepsilon}(t), \xi}\right\|_{P_{g}} \\
& \leq \varepsilon\left\|\operatorname{div}_{g}\left(B\left(\nabla W_{\delta_{\varepsilon}(t), \xi}\right)\right)\right\|_{L^{\frac{2 n}{n+4}}} \\
& \quad+C\left\|f\left(W_{\delta_{\varepsilon}(t), \xi}\right)-P_{g}\left(W_{\delta_{\varepsilon}(t), \xi}\right)\right\|_{L^{\frac{2 n}{n+4}}} \\
& \leq C\left(I_{1}+I_{2}\right) .
\end{aligned}
$$


From (??), we know that $I_{2}=\delta_{\varepsilon}(t)^{2}$. Since

$$
\left\|\frac{1}{r} W_{\delta_{\varepsilon}(t), \xi}^{\prime}\right\|_{L^{\frac{2 n}{n+4}}}+\left\|W_{\delta_{\varepsilon}(t), \xi}^{\prime \prime}\right\|_{L^{\frac{2 n}{n+4}}} \leq O\left(\delta_{\varepsilon}(t)^{2}\left|\ln \delta_{\varepsilon}(t)\right|^{\frac{3}{4}}\right),
$$

we deduce that

$$
\left.I_{1}=\varepsilon\left\|\operatorname{div}_{g}\left(B\left(\nabla W_{\delta_{\varepsilon}(t), \xi}\right)\right)\right\|_{L^{\frac{2 n}{n+4}}}=O\left(\varepsilon \delta_{\varepsilon}(t)^{2}\left|\ln \delta_{\varepsilon}(t)\right|^{\frac{3}{4}}\right)\right) .
$$

The proof follows from the previous estimates.

\subsection{The reduced problem.}

In this section, we will solve equation (??) and give the proof of Theorems ?? and ??. We begin with the case $f_{\varepsilon}(u)=|u|^{2^{*}-2-\varepsilon} u$ (i.e. equation (??)). For $\varepsilon>0$ small enough, we define the energy associated to (??) by, for $u \in H^{2}(M)$,

$$
\begin{aligned}
J_{\varepsilon}(u)= & \frac{1}{2} \int_{M}\left(\Delta_{g} u\right)^{2}+\frac{1}{2} \int_{M} A_{g}\left(\nabla_{g} u, \nabla_{g} u\right) d V+\frac{1}{2} \int_{M} h u^{2} d V \\
& -\int_{M} F_{\varepsilon}(u) d V
\end{aligned}
$$

where $F_{\varepsilon}(u)=\int_{0}^{u} f_{\varepsilon}(s) d s$. We set $I_{\varepsilon}(t, \xi)=J_{\varepsilon}\left(W_{\delta_{\varepsilon}(t), \xi}+\phi_{\delta_{\varepsilon}(t), \xi}\right), t \in \mathbb{R}_{+}^{*}$ and $\xi \in M$ where $\phi_{\delta_{\varepsilon}(t), \xi} \in K_{\delta_{\varepsilon}(t), \xi}^{\perp}$ is the function defined in Proposition ??. In the next proposition, we give the expansion of $I_{\varepsilon}$ with respect to $\varepsilon$.

Proposition 3.4. Assume that $n \geq 8$ and $f_{\varepsilon}(u)=|u|^{2^{*}-2-\varepsilon} u$. There exist constants $c_{i}(n), i=1, \ldots, 5$ depending on $n$, such that

$$
I_{\varepsilon}(t, \xi)=c_{5}(n)+c_{2}(n) \varepsilon+c_{3}(n) \varepsilon \ln \varepsilon-c_{4}(n) \varepsilon \ln (t)+c_{1}(n) \Phi(\xi) \varepsilon t+o(\varepsilon)
$$

as $\varepsilon \rightarrow 0, C^{0}$ uniformly with respect to $t$ in compact subsets of $\mathbb{R}_{+}^{*}$ and with respect to $\xi \in M$ and $C^{1}$ uniformly if $n \geq 11$. Moreover, we have that $c_{4}(n)>0, c_{1}(n)>0$ and

$$
\Phi=-\frac{n^{2}-4 n-4}{96(n-1)(n-3)}\left|W e y l_{g}\right|_{g}^{2}+\frac{1}{n-4}\left(h-\frac{n-4}{2} Q_{g}\right) .
$$

Proof. We begin by proving that

$$
I_{\varepsilon}(t, \xi)=J_{\varepsilon}\left(W_{\delta_{\varepsilon}(t), \xi}\right)+o(\varepsilon),
$$


as $\varepsilon \rightarrow 0$, uniformly with respect to $t$ in compact subsets of $\mathbb{R}_{+}^{*}$ and points $\xi \in M$ (we will show in Lemma ?? that this estimate holds $C^{1}$ uniformly with respect to $t$ and $\xi$ when $n \geq 12$ ). Indeed, we have

$$
\begin{aligned}
& I_{\varepsilon}(t, \xi)-J_{\varepsilon}\left(W_{\delta_{\varepsilon}(t), \xi}\right) \\
& \quad=\left\langle W_{\delta_{\varepsilon}(t), \xi}-i^{*}\left(f_{\varepsilon}\left(W_{\delta_{\varepsilon}(t), \xi}\right)\right), \phi_{\delta_{\varepsilon}(t), \xi}\right\rangle_{P_{g}}+O\left(\left\|\phi_{\delta_{\varepsilon}(t), \xi}\right\|_{P_{g}}^{2}\right)
\end{aligned}
$$

when $\varepsilon \rightarrow 0$. Using Lemma ??, Proposition ?? and the definition of $\delta_{\varepsilon}(t)$ (??), we get

$$
\begin{aligned}
\left\langle W_{\delta_{\varepsilon}(t), \xi}-i^{*}\left(f_{\varepsilon}\left(W_{\delta_{\varepsilon}(t), \xi}\right)\right), \phi_{\delta_{\varepsilon}(t), \xi}\right\rangle_{P_{g}}+O\left(\left\|\phi_{\delta_{\varepsilon}(t), \xi}\right\|_{P_{g}}^{2}\right) \\
=O\left(\left\|\phi_{\delta_{\varepsilon}(t), \xi}\right\|_{P_{g}}^{2}\right) \\
=O\left(\varepsilon^{2}\left|\ln \delta_{\varepsilon}(t)\right|^{2}+\left\{\begin{array}{ll}
\delta_{\varepsilon}(t)^{n-4} & \text { if } n<12 \\
\delta_{\varepsilon}(t)^{8}\left|\ln \delta_{\varepsilon}(t)\right|^{2} & \text { if } n \geq 12
\end{array}\right)\right. \\
=O(\varepsilon) .
\end{aligned}
$$

Now, the proposition is reduced to estimate $J_{\varepsilon}\left(W_{\delta_{\varepsilon}(t), \xi}\right)$. We will focus on $C^{0}$-estimates. The $C^{1}$-estimates can be obtained using the same argument as in Lemma 4.1 of [?]. We use the computations of section 6 of [?] and the estimate (4.2) of [?] to estimate $I_{1, \varepsilon, t, \delta}$. Using that $J_{\varepsilon}$ is conformally invariant and using (??), we have

$$
\begin{aligned}
& \int_{M} P_{g}\left(W_{\delta_{\varepsilon}(t), \xi}\right) W_{\delta_{\varepsilon}(t), \xi} d V=\alpha_{n}^{2}\left[\frac{n(n-4)\left(n^{2}-4\right) \omega_{n}}{2^{n}}\right] \\
& \quad+ \begin{cases}\frac{\omega_{n}(n-1)(n-3)(n-4) \Phi(\xi)}{2^{n-4}(n-6)(n-8)} \delta_{\varepsilon}(t)^{4}+o\left(\delta_{\varepsilon}(t)^{4}\right), & \text { if } n \geq 9, \\
\omega_{n-1}(n-4) \Phi(\xi) \delta_{\varepsilon}(t)^{4}\left|\ln \delta_{\varepsilon}(t)\right|+o\left(\delta_{\varepsilon}(t)^{4}\left|\ln \delta_{\varepsilon}(t)\right|\right), & \text { if } n=8,\end{cases}
\end{aligned}
$$

where $\Phi$ is given by

$$
\Phi=-\frac{n^{2}-4 n-4}{96(n-1)(n-3)}\left|W e y l_{g}\right|_{g}^{2}+\frac{1}{n-4}\left(h-\frac{n-4}{2} Q_{g}\right) .
$$

Next, we define $I_{p}^{q}=\int_{0}^{\infty} \frac{r^{q}}{(1+r)^{p}} d r$ for any $p, q$ integers such that $q<p-1$. In the sequel, we will use that

$$
I_{p}^{q}=\frac{q}{p-q-1} I_{p}^{q-1}=\frac{p}{p-q-1} I_{p+1}^{q},
$$

and

$$
I_{n}^{n-2}=\frac{1}{n-1}, I_{n}^{\frac{n-2}{2}}=\frac{\omega_{n}}{2^{n-1} \omega_{n-1}} .
$$


We also have that

$$
I_{n-\frac{n-4}{2} \varepsilon}^{\frac{n-2}{2}}=I_{n}^{\frac{n-2}{2}}+\frac{n-4}{2} \tilde{I}_{n}^{\frac{n-2}{2}} \varepsilon+O\left(\varepsilon^{2}\right),
$$

where $\tilde{I}_{p}^{q}=\int_{0}^{\infty} \frac{r^{q} \ln (1+r)}{(1+r)^{p}} d r$. Therefore, we obtain that

$$
\begin{aligned}
\frac{1}{2^{*}-\varepsilon} & \int_{M} W_{\delta_{\varepsilon}(t), \xi}^{2^{*}-\varepsilon} d V \\
= & \frac{\alpha_{n}^{2^{*}-\varepsilon}}{2^{*}-\varepsilon}\left(\delta_{\varepsilon}(t)\right)^{\frac{n-4}{2} \varepsilon} \omega_{n-1} \int_{0}^{\frac{r_{0}}{\delta_{\varepsilon}(t)}}\left(\frac{1}{1+r^{2}}\right)^{n-\frac{n-4}{2} \varepsilon} r^{n-1}\left(1+O\left(r^{n}\right)\right) d r \\
= & \frac{n-4}{2 n} K_{n}^{-n / 4}\left[1+\frac{n-4}{2} \varepsilon \ln \left(\delta_{\varepsilon}(t)\right)\right. \\
& \left.+\frac{n-4}{2 n}\left(\frac{n \tilde{I}_{n}^{\frac{n-2}{2}}}{I_{n}^{\frac{n-2}{2}}}+\frac{n\left(1-\frac{n}{2} \ln \sqrt{n(n-4)\left(n^{2}-4\right)}\right)}{n-2}\right) \varepsilon\right]+o\left(\delta_{\varepsilon}(t)^{5}\right) .
\end{aligned}
$$

Then the proposition follows from (??) and (??).

Next we consider equation (??). For $\varepsilon>0$ small enough, we define the energy associated to (??) by, for $u \in H^{2}(M)$,

$$
\begin{aligned}
\tilde{J}_{\varepsilon}(u)= & \frac{1}{2} \int_{M}\left(\Delta_{g} u\right)^{2}+\frac{1}{2} \int_{M}\left(A_{g}+\varepsilon B\right)\left(\nabla_{g} u, \nabla_{g} u\right) d V+\frac{1}{2} \int_{M} h u^{2} d V \\
& -\int_{M} F_{0}(u) d V
\end{aligned}
$$

where $F_{0}(u)=\int_{0}^{u} f_{0}(s) d s$. We set $\tilde{I}_{\varepsilon}(t, \xi)=\tilde{J}_{\varepsilon}\left(W_{\delta_{\varepsilon}(t), \xi}+\phi_{\delta_{\varepsilon}(t), \xi}\right), t \in \mathbb{R}_{+}^{*}$ and $\xi \in M$ where $\phi_{\delta_{\varepsilon}(t), \xi} \in K_{\delta_{\varepsilon}(t), \xi}^{\perp}$ is the function defined in Proposition ??. In this case, we obtain :

Proposition 3.5. Assume that $n=8$ and $f_{\varepsilon}(u)=|u|^{2^{*}-2} u-\varepsilon d i v_{g}(B(\nabla u))$. There exist constants $c_{i}(n), i=1, \ldots, 3$ depending on $n$, such that

$$
\begin{aligned}
\tilde{I}_{\varepsilon}(t, \xi)= & c_{1}(n)+\varepsilon^{2}|\ln \varepsilon|^{-1}\left(c_{2}(n) t^{2} \operatorname{Tr}_{g} B(\xi)-c_{3}(n) t^{4}\left|W e y l_{g}\right|_{g}^{2}\right) \\
& +o\left(\varepsilon^{2}|\ln \varepsilon|^{-1}\right),
\end{aligned}
$$

as $\varepsilon \rightarrow 0 C^{0}$ uniformly with respect to $t$ in compact subsets of $\mathbb{R}_{+}^{*}$ and with respect to $\xi \in M$. Moreover, we have that $c_{2}(n)>0$ and $c_{3}(n)>0$. 
Proof. Using Lemma ??, Proposition ?? and (??), we get

$$
\begin{aligned}
& \left\langle W_{\delta_{\varepsilon}(t), \xi}-i^{*}\left(f_{\varepsilon}\left(W_{\delta_{\varepsilon}(t), \xi}\right)\right), \phi_{\delta_{\varepsilon}(t), \xi}\right\rangle_{P_{g}} \\
& +O\left(\left\|\phi_{\delta_{\varepsilon}(t), \xi}\right\|_{P_{g}}^{2}\right)=O\left(\delta_{\varepsilon}(t)^{4}\right) .
\end{aligned}
$$

We have

$$
\frac{1}{2^{*}} \int_{M} W_{\delta_{\varepsilon}(t), \xi}^{2^{*}} d V=\frac{\omega_{n}}{2^{n}}+o\left(\delta_{\varepsilon}(t)^{5}\right)
$$

and

$$
\begin{aligned}
\frac{1}{2} \varepsilon \int_{M} B & \left(\nabla W_{\delta_{\varepsilon}(t), \xi}, \nabla W_{\delta_{\varepsilon}(t), \xi}\right) d V \\
\quad= & \frac{(n-4)^{2}}{2 n} \varepsilon \delta_{\varepsilon}(t)^{2} \alpha_{n}^{2} \omega_{n-1} \operatorname{Tr}_{g} B(\xi) \int_{0}^{\frac{r_{0}}{\delta_{\varepsilon}(t)}} \frac{r^{n+1}}{\left(1+r^{2}\right)^{n-2}} d r+O\left(\delta_{\varepsilon}(t)^{5}\right) \\
= & \varepsilon \delta_{\varepsilon}(t)^{2} \frac{2(n-1)}{n(n-6)\left(n^{2}-4\right)} K_{n}^{-\frac{n}{4}} \operatorname{Tr}_{g} B(\xi)+O\left(\delta_{\varepsilon}(t)^{5}\right) .
\end{aligned}
$$

Therefore, from the two previous estimates and (??), we have

$$
\begin{aligned}
& \tilde{J}_{\varepsilon}\left(W_{\delta_{\varepsilon}(t), \xi}\right)= \alpha_{n}^{2}\left[\frac{n(n-4)\left(n^{2}-4\right) \omega_{n}}{2^{n}}\right] \\
&+\varepsilon \delta_{\varepsilon}(t)^{2} \frac{2(n-1)}{n(n-6)\left(n^{2}-4\right)} K_{n}^{-\frac{n}{4}} \operatorname{Tr}_{g} B(\xi) \\
&-\frac{n^{2}-4 n-4}{96(n-1)(n-3)}\left|W e y l_{g}\right|_{g}^{2} \delta_{\varepsilon}(t)^{4}\left|\ln \delta_{\varepsilon}(t)\right|+o\left(\delta_{\varepsilon}(t)^{4}\left|\ln \delta_{\varepsilon}(t)\right|\right) .
\end{aligned}
$$

Finally, we recall the following proposition (see [?] and [?] for a proof) which shows that to obtain a solution of (??) (respectively (??)), we only need to find a critical point for $\tilde{I}_{\varepsilon}$ (respectively $I_{\varepsilon}$ ).

Proposition 3.6. Given two positive real numbers $a<b$, for $\varepsilon$ small, if $\left(t_{\varepsilon}, \xi_{\varepsilon}\right) \in(a, b) \times M$ is a critical point of $\tilde{I}_{\varepsilon}$ (respectively $\left.I_{\varepsilon}\right)$, then the function $W_{\delta_{\varepsilon}\left(t_{\varepsilon}\right), \xi_{\varepsilon}}+\phi_{\delta_{\varepsilon}\left(t_{\varepsilon}\right), \xi_{\varepsilon}}$ is a solution of (??) (respectively (??)).

We are now in position to prove Theorems ?? and ??. We restrict ourselves to prove Theorem ?? (Theorem ?? can be obtained in the same way).

Proof of Theorem ??. We set $\mathcal{G}: \mathbb{R}_{+}^{*} \times M \rightarrow \mathbb{R}$ the function defined by

$$
\mathcal{G}(t, \xi)=-c_{4}(n) \ln t+c_{1}(n) \varphi(\xi) t
$$


where $c_{4}(n), c_{1}(n)$ and $\varphi(\xi)$ are defined in (??). From Proposition ??, we have

$$
\lim _{\varepsilon \rightarrow 0} \frac{1}{\varepsilon}\left(I_{\varepsilon}(t, \xi)-c_{5}(n)-c_{2}(n) \varepsilon-c_{3}(n) \varepsilon \ln \varepsilon\right)=\mathcal{G}(t, \xi),
$$

$C^{0}$ (and $C^{1}$ if $n \geq 11$ ) uniformly with respect to $\xi \in M$ and $t$ in compact subset of $\mathbb{R}_{+}^{*}$. We will consider two cases depending on the dimension of the manifold.

First case $: n \geq 11$.

We argue as in [?]. Let $\xi_{0}$ be the $C^{1}$ stable critical point of $\varphi$ such that $\varphi\left(\xi_{0}\right)>0$ and set

$$
t_{0}=\frac{c_{4}(n)}{c_{1}(n) \varphi\left(\xi_{0}\right)}>0 .
$$

Identifying the tangent space at $\xi$ with $\mathbb{R}^{n}$ we define the map $H$ from $[0,1] \times$ $\mathbb{R}^{+} \times \mathbb{R}^{n}$ into $\mathbb{R}^{n+1}$ by

$$
\begin{aligned}
H(s, t, \xi)=s & \left(\frac{\partial \mathcal{G}\left(t, \exp _{\xi}(y)\right)}{\partial t},\left.\frac{\partial \mathcal{G}\left(t, \exp _{\xi}(y)\right)}{\partial y_{1}}\right|_{y=0}, \ldots,\left.\frac{\partial \mathcal{G}\left(t, \exp _{\xi}(y)\right)}{\partial y_{n}}\right|_{y=0}\right) \\
& +(1-s)\left(t-t_{0},\left.\frac{\partial\left(\varphi \circ \exp _{\xi}(y)\right)}{\partial y_{1}}\right|_{y=0}, \ldots,\left.\frac{\partial\left(\varphi \circ \exp _{\xi}(y)\right)}{\partial y_{n}}\right|_{y=0}\right)
\end{aligned}
$$

By the invariance of the Brower degree via homotopy, we have that $\left(t_{0}, \xi_{0}\right)$ is a $C^{1}$ stable critical point of $\mathcal{G}$. From Proposition ?? and standard properties of the Brower degree (see e.g. [?]), there exists a couple $\left(t_{\varepsilon}, \xi_{\varepsilon}\right)$ of critical points of $I_{\varepsilon}$ converging to $\left(t_{0}, \xi_{0}\right)$.

Second case : $8 \leq n \leq 10$.

Since $c_{4}(n)$ and $c_{1}(n)$ are positive, we have

$$
\lim _{t \rightarrow 0^{+}} \mathcal{G}(t, \xi)=\lim _{t \rightarrow \infty} \mathcal{G}(t, \xi)=+\infty,
$$

uniformly in $\xi \in M$. Therefore, from (??) we deduce that, for $\varepsilon$ small enough,there exists a couple $\left(t_{\varepsilon}, \xi_{\varepsilon}\right)$ which is a minimum for the functional $I_{\varepsilon}$ in $(a, b) \times M$ where $a, b$ are positive constants not depending on $\varepsilon$. This implies from Proposition ?? that $W_{\delta_{\varepsilon}\left(t_{\varepsilon}\right), \xi_{\varepsilon}}+\phi_{\delta_{\varepsilon}\left(t_{\varepsilon}\right), \xi_{\varepsilon}}$ is a solution of (??). Thus Theorem ?? is established.

\section{3 $C^{1}$ uniform estimate for the reduced energy.}

Finally, we end this section by proving that the estimate (??) holds $C^{1}$ uniformly if $n \geq 11$. 
Lemma 3.7. If $n \geq 11$, we have

$$
I_{\varepsilon}(t, \xi)=J_{\varepsilon}\left(W_{\delta_{\varepsilon}(t), \xi}\right)+o(\varepsilon),
$$

$C^{1}$ uniformly with respect to $t$ in compact subsets of $\mathbb{R}_{+}^{*}$ and $\xi \in M$.

Proof. To simplify notations, we set, for $i=1, \ldots, n$,

$$
Z_{0}=Z_{\delta_{\varepsilon}(t), \xi} \text { and } Z_{i}=Z_{\delta_{\varepsilon}(t), \xi, e_{i}} \text {. }
$$

We recall that

$$
\frac{\partial}{\partial t}\left(W_{\delta_{\varepsilon}(t), \xi}\right)=\frac{\tilde{C}_{n}}{t} Z_{0}=\frac{\tilde{C}_{n} \delta_{\varepsilon}^{\prime}(t)}{\delta_{\varepsilon}(t)} Z_{0}
$$

where $\tilde{C}_{n}=\frac{\alpha_{n}(n-4)}{4}\left(\right.$ see (??) for the definition of $\left.\alpha_{n}\right)$. Taking the derivative with respect to $t$ to $I_{\varepsilon}(t, \xi)-J_{\varepsilon}\left(W_{\delta_{\varepsilon}(t), \xi}\right)$, we obtain

$$
\begin{aligned}
\frac{\partial I_{\varepsilon}}{\partial t}(t, \xi) & -\frac{\partial J_{\varepsilon}}{\partial t}\left(W_{\delta(t), \xi}\right) \\
= & \int_{M} P_{g}\left(\phi_{\delta_{\varepsilon}(t), \xi}\right) \frac{\partial}{\partial t} W_{\delta_{\varepsilon}(t), \xi} d V \\
& -\int_{M}\left(f_{\varepsilon}\left(W_{\delta_{\varepsilon}(t), \xi}+\phi_{\delta_{\varepsilon}(t), \xi}\right)-f_{\varepsilon}\left(W_{\delta_{\varepsilon}(t), \xi}\right)\right) \frac{\partial W_{\delta_{\varepsilon}(t), \xi}}{\partial t} d V \\
& +D J_{\varepsilon}\left(W_{\delta_{\varepsilon}(t), \xi}+\phi_{\delta_{\varepsilon}(t), \xi}\right)\left[\frac{\partial \phi_{\delta_{\varepsilon}(t), \xi}}{\partial t}\right] \\
= & \frac{\tilde{C}_{n}}{t}\left(\int_{M}\left(P_{g}\left(Z_{0}\right)-f_{\varepsilon}^{\prime}\left(W_{\delta_{\varepsilon}(t), \xi}\right) Z_{0}\right) \phi_{\delta_{\varepsilon}(t), \xi} d V\right. \\
& -\int_{M}\left(f_{\varepsilon}\left(W_{\delta_{\varepsilon}(t), \xi}+\phi_{\delta_{\varepsilon}(t), \xi}\right)-f_{\varepsilon}\left(W_{\delta_{\varepsilon}(t), \xi}\right)\right. \\
& \left.\left.+D J_{\varepsilon}\left(W_{\delta_{\varepsilon}(t), \xi}\right) \phi_{\delta_{\varepsilon}(t), \xi}\right) Z_{0} d V\right) \\
= & I_{1}+I_{2}+I_{\delta_{\varepsilon}(t), \xi},\left[\frac{\partial \phi_{\delta_{\varepsilon}(t), \xi}}{\partial t}\right]
\end{aligned}
$$


where

$$
\begin{aligned}
I_{1}= & \frac{\tilde{C}_{n}}{t} \int_{M}\left(P_{g}\left(Z_{0}\right)-f_{\varepsilon}^{\prime}\left(W_{\delta_{\varepsilon}(t), \xi}\right) Z_{0}\right) \phi_{\delta_{\varepsilon}(t), \xi} d V \\
I_{2}= & -\frac{\tilde{C}_{n}}{t} \int_{M}\left(f_{\varepsilon}\left(W_{\delta_{\varepsilon}(t), \xi}+\phi_{\delta_{\varepsilon}(t), \xi}\right)-f_{\varepsilon}\left(W_{\delta_{\varepsilon}(t), \xi}\right)\right. \\
& \left.-f_{\varepsilon}^{\prime}\left(W_{\delta_{\varepsilon}(t), \xi}\right) \phi_{\delta_{\varepsilon}(t), \xi}\right) Z_{0} d V \\
I_{3}= & D J_{\varepsilon}\left(W_{\delta_{\varepsilon}(t), \xi}+\phi_{\delta_{\varepsilon}(t), \xi}\right)\left[\frac{\partial \phi_{\delta_{\varepsilon}(t), \xi}}{\partial t}\right]
\end{aligned}
$$

In the same way, recalling that

$$
\left.\frac{\partial}{\partial y_{i}}\left(W_{\delta_{\varepsilon}(t), \exp _{\xi}(y)}\right)\right|_{y=0}=\frac{\alpha_{n}(n-4)}{\delta_{\varepsilon}(t)} Z_{i}+R_{\delta_{\varepsilon}(t), \xi},
$$

where $\left\|R_{\delta_{\varepsilon}(t), \xi}\right\|_{P_{g}}=O\left(\delta_{\varepsilon}(t)^{2}\right)$ (see (6.13) of [?]) and using (??), we find

$$
\begin{aligned}
& \frac{\partial I_{\varepsilon}}{\partial y_{i}}\left(t,\left.\exp _{\xi}(y)\right|_{y=0}-\left.\frac{\partial J_{\varepsilon}}{\partial y_{i}}\left(W_{\delta(t), \exp _{\xi}(y)}\right)\right|_{y=0}\right. \\
& =\frac{\alpha_{n}(n-4)}{\delta_{\varepsilon}(t)}\left(\int_{M}\left(P_{g}\left(Z_{i}\right)-f_{\varepsilon}^{\prime}\left(W_{\delta_{\varepsilon}(t), \xi}\right) Z_{i}\right) \phi_{\delta_{\varepsilon}(t), \xi} d V\right. \\
& -\int_{M}\left(f_{\varepsilon}\left(W_{\delta_{\varepsilon}(t), \xi}+\phi_{\delta_{\varepsilon}(t), \xi}\right)-f_{\varepsilon}\left(W_{\delta_{\varepsilon}(t), \xi}\right)\right. \\
& \left.\left.-f_{\varepsilon}^{\prime}\left(W_{\delta_{\varepsilon}(t), \xi}\right) \phi_{\delta_{\varepsilon}(t), \xi}\right) Z_{i} d V\right) \\
& +D J_{\varepsilon}\left(W_{\delta_{\varepsilon}(t), \xi}+\phi_{\delta_{\varepsilon}(t), \xi}\right)\left[\frac{\partial \phi_{\delta_{\varepsilon}(t), \exp _{\xi}(y)}}{\partial y_{i}}\right]_{y=0} \\
& +O\left(\left\|R_{\delta_{\varepsilon}(t), \xi}\right\|_{P_{g}}\left\|\phi_{\delta_{\varepsilon}(t), \xi}\right\|_{P_{g}}\right) \\
& =I_{4}+I_{5}+I_{6}+o(\varepsilon)
\end{aligned}
$$

where

$$
\begin{gathered}
I_{4}=\frac{\alpha_{n}(n-4)}{\delta_{\varepsilon}(t)} \int_{M}\left(P_{g}\left(Z_{i}\right)-f_{\varepsilon}^{\prime}\left(W_{\delta_{\varepsilon}(t), \xi}\right) Z_{i}\right) \phi_{\delta_{\varepsilon}(t), \xi} d V \\
I_{5}=-\frac{\alpha_{n}(n-4)}{\delta_{\varepsilon}(t)} \int_{M}\left(f_{\varepsilon}\left(W_{\delta_{\varepsilon}(t), \xi}+\phi_{\delta_{\varepsilon}(t), \xi}\right)-f_{\varepsilon}\left(W_{\delta_{\varepsilon}(t), \xi}\right)\right. \\
\left.-f_{\varepsilon}^{\prime}\left(W_{\delta_{\varepsilon}(t), \xi}\right) \phi_{\delta_{\varepsilon}(t), \xi}\right) Z_{i} d V \\
I_{6}=D J_{\varepsilon}\left(W_{\delta_{\varepsilon}(t), \xi}+\phi_{\delta_{\varepsilon}(t), \xi}\right)\left[\frac{\partial \phi_{\delta_{\varepsilon}(t), \exp _{\xi}(y)}}{\partial y_{i}}\right]_{y=0} .
\end{gathered}
$$


We begin by estimating the terms $I_{3}$ and $I_{6}$. By Proposition ??, there exist real numbers $\lambda_{i}, i=0, \ldots, n$ such that

$$
D J_{\varepsilon}\left(W_{\delta_{\varepsilon}(t), \xi}+\phi_{\delta_{\varepsilon}(t), \xi}\right)[.]=\sum_{i=0}^{n} \lambda_{i}\left\langle Z_{i}, .\right\rangle_{P_{g}} .
$$

Arguing in the same way as in Proposition 2.2 of [?] (see in particular (4.23) and (4.24)), we have

$$
D J\left(W_{\delta_{\varepsilon}(t), \xi}+\phi_{\delta_{\varepsilon}(t), \xi}\left[\frac{\partial \phi_{\delta_{\varepsilon}(t), \xi}}{\partial t}\right]=O\left(\left\|\phi_{\delta_{\varepsilon}(t), \xi}\right\|_{L^{\frac{2 n}{n+4}}} \sum_{i=0}^{n}\left|\lambda_{i}\right|\right),\right.
$$

and

$$
D J_{\varepsilon}\left(W_{\delta_{\varepsilon}(t), \xi}+\phi_{\delta_{\varepsilon}(t), \xi}\left[\frac{\partial \phi_{\delta_{\varepsilon}(t), \exp _{\xi}(y)}}{\partial y_{i}}\right]_{\mid y=0}=O\left(\frac{\left\|\phi_{\delta_{\varepsilon}(t), \xi}\right\|_{L^{\frac{2 n}{n+4}} \sum_{i=0}^{n}\left|\lambda_{i}\right|}}{\delta_{\varepsilon}(t)}\right) .\right.
$$

We claim that $\left|\lambda_{i}\right|=O\left(\left\|P_{g}\left(W_{\delta_{\varepsilon}(t), \xi}\right)-f_{\varepsilon}\left(W_{\delta_{\varepsilon}(t), \xi}\right)\right\|_{\frac{2 n}{n+4}}\right)$, for all $i=0, \ldots, n$. Using that

$$
\left\langle Z_{i}, Z_{j}\right\rangle_{P_{g}} \rightarrow\left\|\Delta_{\text {eucl }} V_{i}\right\|_{L^{2}\left(\mathbb{R}^{n}\right)}^{2} \delta_{i j},
$$

to prove the claim, we just need to show that

$$
D J\left(W_{\delta_{\varepsilon}(t), \xi}+\phi_{\delta_{\varepsilon}(t), \xi}\right)\left[Z_{i}\right]=O\left(\left\|P_{g}\left(W_{\delta_{\varepsilon}(t), \xi}\right)-f_{\varepsilon}\left(W_{\delta_{\varepsilon}(t), \xi}\right)\right\|_{L^{\frac{2 n}{n+4}}}\right),
$$

for all $i=0, \ldots, n$. Since $\phi_{\delta_{\varepsilon}(t), \xi} \in K_{\delta_{\varepsilon}(t), \xi}^{\perp}$, using Hölder inequality, (??), Lemma ?? and rough estimates, we have

$$
\begin{aligned}
D J_{\varepsilon}\left(W_{\delta_{\varepsilon}(t), \xi}\right. & \left.+\phi_{\delta_{\varepsilon}(t), \xi}\right)\left[Z_{i}\right] \\
= & \int_{M} P_{g}\left(W_{\delta_{\varepsilon}(t), \xi}\right) Z_{i} d V-\int_{M} f_{\varepsilon}\left(W_{\delta_{\varepsilon}(t), \xi}+\phi_{\delta_{\varepsilon}(t), \xi}\right) Z_{i} d V \\
= & \int_{M}\left(P_{g}\left(W_{\delta_{\varepsilon}(t), \xi}\right)-f_{\varepsilon}\left(W_{\delta_{\varepsilon}(t), \xi}\right)\right) Z_{i} d V \\
& -\int_{M}\left(f_{\varepsilon}\left(W_{\delta_{\varepsilon}(t), \xi}+\phi_{\delta_{\varepsilon}(t), \xi}\right)-f_{\varepsilon}\left(W_{\delta_{\varepsilon}(t), \xi}\right)\right) Z_{i} d V \\
\leq & \left\|P_{g}\left(W_{\delta_{\varepsilon}(t), \xi}\right)-f_{\varepsilon}\left(W_{\delta_{\varepsilon}(t), \xi}\right)\right\|_{L^{\frac{2 n}{n+4}}}\left\|Z_{i}\right\|_{L^{2^{*}}} \\
& +\left\|f_{\varepsilon}\left(W_{\delta_{\varepsilon}(t), \xi}+\phi_{\delta_{\varepsilon}(t), \xi}\right)-f_{\varepsilon}\left(W_{\delta_{\varepsilon}(t), \xi}\right)\right\|_{L^{\frac{2 n}{n+4}}}\left\|Z_{i}\right\|_{L^{2^{*}}} \\
\leq & O\left(\left\|P_{g}\left(W_{\delta_{\varepsilon}(t), \xi}\right)-f_{\varepsilon}\left(W_{\delta_{\varepsilon}(t), \xi}\right)\right\|_{L^{\frac{2 n}{n+4}}}\right) \\
& +O\left(\left\|\phi_{\delta_{\varepsilon}(t), \xi}\right\|_{L^{\frac{2 n}{n-4}}}\left(\left\|W_{\delta_{\varepsilon}(t), \xi}\right\|_{L^{\frac{2 n}{n-4}}}^{2^{*}-2-\varepsilon}+\left\|\phi_{\delta_{\varepsilon}(t), \xi}\right\|_{L^{\frac{2 n}{n-4}}}^{2^{*}-2-\varepsilon}\right)\right) \\
\leq & O\left(\left\|P_{g}\left(W_{\delta_{\varepsilon}(t), \xi}\right)-f_{\varepsilon}\left(W_{\delta_{\varepsilon}(t), \xi}\right)\right\|_{L^{\frac{2 n}{n+4}}}\right) .
\end{aligned}
$$


Combining the previous estimates, we get

$$
\begin{aligned}
D J_{\varepsilon}\left(W_{\delta_{\varepsilon}(t), \xi}+\phi_{\delta_{\varepsilon}(t), \xi}\right)\left[\frac{\partial \phi_{\delta_{\varepsilon}(t), \xi}}{\partial t}\right] & \\
= & O\left(\left\|P_{g}\left(W_{\delta_{\varepsilon}(t), \xi}\right)-f_{\varepsilon}\left(W_{\delta_{\varepsilon}(t), \xi}\right)\right\|_{L^{\frac{2 n}{n+4}}}^{2}\right),
\end{aligned}
$$

and

$$
\begin{aligned}
D J_{\varepsilon}\left(W_{\delta_{\varepsilon}(t), \xi}+\phi_{\delta_{\varepsilon}(t), \xi}\right) & {\left.\left[\frac{\partial \phi_{\delta_{\varepsilon}(t), \exp _{\xi}(y)}}{\partial y_{i}}\right]\right|_{y=0} } \\
& =O\left(\frac{\left\|P_{g}\left(W_{\delta_{\varepsilon}(t), \xi}\right)-f_{\varepsilon}\left(W_{\delta_{\varepsilon}(t), \xi}\right)\right\|_{L^{\frac{2 n}{n+4}}}^{2}}{\delta_{\varepsilon}(t)}\right) .
\end{aligned}
$$

Now let us estimate $I_{2}$ and $I_{5}$. Noticing that, if $n \geq 11$,

$$
\left\|\left(W_{\delta_{\varepsilon}(t), \xi}\right)^{2^{*}-3-\varepsilon} Z_{i}\right\|_{L^{\frac{n}{4}}}=O(1),
$$

we obtain, for $i=0, \ldots, n$,

$$
\begin{aligned}
& \int_{M}\left(f_{\varepsilon}\left(W_{\delta_{\varepsilon}(t), \xi}+\phi_{\delta_{\varepsilon}(t), \xi}\right)-f_{\varepsilon}\left(W_{\delta_{\varepsilon}(t), \xi}\right)-f_{\varepsilon}^{\prime}\left(W_{\delta_{\varepsilon}(t), \xi}\right) \phi_{\delta_{\varepsilon}(t), \xi}\right) Z_{i} d V \\
& \leq C\left\{\begin{array}{l}
\int_{M}\left(W_{\delta_{\varepsilon}(t), \xi}\right)^{2^{*}-3-\varepsilon} \phi_{\delta_{\varepsilon}(t), \xi}^{2} Z_{i} d V, \\
\int_{M}\left(\left(W_{\delta_{\varepsilon}(t), \xi}\right)^{2^{*}-3-\varepsilon} \phi_{\delta_{\varepsilon}(t), \xi}^{2}+\phi_{\delta_{\varepsilon}(t), \xi}^{2^{*}-1-\varepsilon} Z_{i} d V\right), \quad \text { if } n \geq 12,
\end{array}\right. \\
& \leq C\left\{\begin{array}{c}
\left\|\left(W_{\delta_{\varepsilon}(t), \xi}\right)^{2^{*}-3-\varepsilon} Z_{i}\right\|_{L^{\frac{n}{4}}}\left\|\phi_{\delta_{\varepsilon}(t), \xi}\right\|_{L^{\frac{2 n}{n-4}}}^{2}, \quad \text { if } n \geq 11, \\
\left\|\phi_{\delta_{\varepsilon}(t), \xi}\right\|_{L^{\frac{2 n}{n-4}}}^{2}\left\|\left(W_{\delta_{\varepsilon}(t), \xi}\right)^{2^{*}-3-\varepsilon} Z_{i}\right\|_{L^{\frac{n}{4}}} \\
+\left\|Z_{i}\right\|_{L^{\frac{2 n}{n-4}}}\left\|\phi_{\delta_{\varepsilon}(t), \xi}\right\|_{L^{\frac{2 n}{n-4}}}^{2^{*}-1-\varepsilon},
\end{array} \quad \text { if } n=11,\right. \\
& \leq O\left(\left\|\phi_{\delta_{\varepsilon}(t), \xi}\right\|_{L^{\frac{2 n}{n-4}}}^{2}\right) \text { when } n \geq 11 .
\end{aligned}
$$

Finally, let us estimate $I_{1}$ and $I_{4}$. Since

$$
\left\|P_{g}\left(Z_{i}\right)-f_{\varepsilon}^{\prime}\left(W_{\delta_{\varepsilon}(t), \xi}\right) Z_{i}\right\|_{L^{\frac{2 n}{n+4}}}=O\left(\delta_{\varepsilon}(t)^{2}\right)
$$

(one can argue as in [?], inequality (4.17)), we obtain

$$
\begin{aligned}
\int_{M}\left(P_{g}\left(Z_{i}\right)-f_{\varepsilon}^{\prime}\left(W_{\delta_{\varepsilon}(t), \xi}\right) Z_{i}\right) & \phi_{\delta_{\varepsilon}(t), \xi} d V \\
& \leq C\left\|P_{g}\left(Z_{i}\right)-f_{\varepsilon}^{\prime}\left(W_{\delta_{\varepsilon}(t), \xi}\right) Z_{i}\right\|_{L^{\frac{2 n}{n+4}}}\left\|\phi_{\delta_{\varepsilon}(t), \xi}\right\|_{L^{2^{*}}} \\
& \leq O\left(\delta_{\varepsilon}(t)^{2}\left\|\phi_{\delta_{\varepsilon}(t), \xi}\right\|_{L^{2^{*}}}\right) .
\end{aligned}
$$

The lemma now follows from (??), (??), (??), (??), (??) and (??). 


\section{Case $M$ is l.c.f. : Proof of Theorem ??.}

In all this section, we assume that $M$ is l.c.f. We will also assume that $G_{g_{\xi}}$, the Green function of $P_{g_{\xi}}$, is of the form

$$
G_{g_{\xi}}\left(\exp _{\xi} y, \xi\right)=\frac{1}{\beta_{n}|y|^{n-4}}+A+0^{(4)}(|y|)
$$

where $\beta_{n}=(n-2)(n-4) \omega_{n-1}$ (see the introduction for the definition of $\left.0^{(4)}(|y|)\right)$. In the following, with an abuse of notation, we will identify the metric $g$ and $g_{\xi}$. In this section, we will modify the notation of the function $W_{\delta_{\varepsilon}(t), \xi}$ defined in the previous one. Here, we will be looking for a solution of the form

$$
W_{\delta_{\varepsilon}(t), \xi}(x)=G_{g}(x, \xi) \hat{W}_{\delta_{\varepsilon}(t), \xi}(x),
$$

where $\hat{W}_{\delta_{\varepsilon}(t), \xi}$ is defined by

$$
\hat{W}_{\delta_{\varepsilon}(t), \xi}=\left\{\begin{array}{cl}
\hat{W}_{\delta_{\varepsilon}(t), \xi}^{\text {in }}(x):=\beta_{n} \delta_{\varepsilon}(t)^{\frac{4-n}{2}} d(x, \xi)^{n-4} U\left(\frac{d(x, \xi)}{\delta_{\varepsilon}(t)}\right), & \text { if } d(x, \xi) \leq r_{0}, \\
\hat{W}_{\delta_{\varepsilon}(t), \xi}^{\text {out }}(x):=\beta_{n} \delta_{\varepsilon}(t)^{\frac{4-n}{2}} r_{0}^{n-4} U\left(\frac{r_{0}}{\delta_{\varepsilon}(t)}\right) & \text { if } d(x, \xi)>r_{0} . \\
+\gamma_{\tilde{\varepsilon}}\left(d(x, \xi)-r_{0}\right)\left(\hat{W}_{\delta_{\varepsilon}(t), \xi}^{\text {in }}\right)^{\prime}\left(r_{0}\right), &
\end{array}\right.
$$

In the previous definition, $\gamma_{\tilde{\varepsilon}}: \mathbb{R} \rightarrow \mathbb{R}, \tilde{\varepsilon} \in \mathbb{R}^{+}$is a smooth function satisfying the following properties :

$$
\begin{gathered}
\operatorname{supp}\left(\gamma_{\tilde{\varepsilon}}\right) \subset[0, \tilde{\varepsilon}], \\
\gamma(0)=0, \gamma^{\prime}(0)=1,\left|\nabla^{i} \gamma(r)\right| \leq \tilde{\varepsilon}^{-i}, \forall r \geq 0 \text { and } i=1, \ldots, 4
\end{gathered}
$$

It is easy to check that $\hat{W}_{\delta_{\varepsilon}(t), \xi} \in H^{2}(M)$. We also define, for any real $\delta$ strictly positive, $\xi \in M$ and $x \in M$,

$$
Z_{\delta, \xi}(x)=G_{g}(x, \xi) \hat{Z}_{\delta, \xi}(x),
$$

and, for $\omega \in T_{\xi} M$,

$$
Z_{\delta, \xi, \omega}(x)=G_{g}(x, \xi) \hat{Z}_{\delta, \xi, \omega}(x)
$$

where

$$
\hat{Z}_{\delta, \xi}(x)=d(x, \xi)^{n-4} \chi\left(d_{g}(x, \xi)\right) \delta^{\frac{n-4}{2}} \frac{d(x, \xi)^{2}-\delta^{2}}{\left(\delta^{2}+d(x, \xi)^{2}\right)^{\frac{n-2}{2}}},
$$

and, for $\omega \in T_{\xi} M$,

$$
\hat{Z}_{\delta, \xi, \omega}(x)=d(x, \xi)^{n-4} \chi\left(d_{g}(x, \xi)\right) \delta^{\frac{n-2}{2}} \frac{\left\langle\exp _{\xi}^{-1} x, \omega\right\rangle_{g}}{\left(\delta^{2}+d(x, \xi)^{2}\right)^{\frac{n-2}{2}}},
$$


$\chi$ is a smooth cut-off function such that $0 \leq \chi \leq 1, \chi \equiv 0$ in $\left[r_{0}, \infty\right)$ and $\chi \equiv 1$ in $\left[0, \frac{r_{0}}{2}\right]$. In this section, we choose

$$
\delta_{\varepsilon}(t)=\left\{\begin{array}{ll}
t \varepsilon^{\frac{1}{n-6}} & \text { if } n \geq 7 \\
e^{-\frac{t}{\varepsilon}} & \text { if } n=6
\end{array} .\right.
$$

In view of the results of the previous section, it is easy to see that we only need to obtain an estimate of the error and of the reduced energy in order to prove Theorem ??. We begin with the error estimate.

Lemma 4.1. Assume that $M$ is l.c.f. and $f_{\varepsilon}(u)=|u|^{2^{*}-2} u-\varepsilon \operatorname{div}_{g}(B(\nabla u))$. Given two positive real numbers $a<b$, there exists a positive constant $C_{a, b}^{\prime}$ such that for $\varepsilon$ small, for any real number $t \in[a, b]$ and any point $\xi \in M$, there holds

$$
\begin{aligned}
& \| i^{*}\left(f_{\varepsilon}\left(W_{\delta_{\varepsilon}(t), \xi}\right)\right)- W_{\delta_{\varepsilon}(t), \xi} \|_{P_{g}} \\
& \leq C_{a, b}^{\prime} \begin{cases}\delta_{\varepsilon}(t)^{n-4}+\varepsilon \delta_{\varepsilon}(t)^{\frac{n-4}{2}}, & \text { if } n<8, \\
\delta_{\varepsilon}(t)^{4}\left|\ln \delta_{\varepsilon}(t)\right|^{\frac{3}{4}}+\varepsilon \delta_{\varepsilon}(t)^{2}\left|\ln \delta_{\varepsilon}(t)\right|^{\frac{3}{4}}, & \text { if } n=8, \\
\delta_{\varepsilon}(t)^{\frac{n}{2}}+\varepsilon \delta_{\varepsilon}(t)^{2} & \text { if } n>8 .\end{cases}
\end{aligned}
$$

Proof. Integrating by parts, we find

$$
\begin{array}{r}
\left\langle i^{*}\left(f_{\varepsilon}\left(W_{\delta_{\varepsilon}(t), \xi}\right)\right)-W_{\delta_{\varepsilon}(t), \xi}, \phi\right\rangle=\int_{M}\left(f_{\varepsilon}\left(W_{\delta_{\varepsilon}(t), \xi}\right)-P_{g} W_{\delta_{\varepsilon}(t), \xi}\right) \phi d V \\
-\int_{\partial B_{\xi}\left(r_{0}\right)}\left(\Delta_{g} W_{\delta_{\varepsilon}(t), \xi}^{\text {out }} \partial_{\text {in }} \phi+\Delta_{g} W_{\delta_{\varepsilon}(t), \xi}^{\text {in }} \partial_{\text {out }} \phi\right) d \sigma \\
\quad+\int_{\partial B_{\xi}\left(r_{0}\right)}\left(\partial_{\text {in }}\left(\Delta_{g} W_{\delta_{\varepsilon}(t), \xi}^{\text {out }}\right)+\partial_{\text {out }}\left(\Delta_{g} W_{\delta_{\varepsilon}(t), \xi}^{\text {in }}\right)\right) \phi d \sigma
\end{array}
$$

where $\partial B_{\xi}\left(r_{0}\right)$ is the boundary of the geodesic ball with respect to $g_{\xi}$ of radius $r_{0}$ centered in $\xi$ and $\partial_{\nu_{\text {in }}}$ (resp. $\partial_{\nu_{\text {out }}}$ ) stands for the derivatives with respect to the inward (resp. outward), unit normal vectors of $\partial B_{\xi}\left(r_{0}\right)$ and $d \sigma_{g}$ is the volume element of $\partial B_{\xi}\left(r_{0}\right)$. Now, straight forward computations give, on $\partial B_{\xi}\left(r_{0}\right)$,

$$
\begin{gathered}
\left(\partial_{\text {in }} \Delta_{g} W_{\delta_{\varepsilon}(t), \xi}^{\text {out }}+\partial_{\text {out }} \Delta_{g} W_{\delta_{\varepsilon}(t), \xi}^{\text {in }}\right) \\
+G_{g}\left(\left(\Delta_{g} \hat{W}_{\delta_{\varepsilon}(t), \xi}^{\text {in }}\right)^{\prime}-\left(\Delta_{g} \hat{W}_{\delta_{\varepsilon}(t), \xi}^{\text {out }}\left(\Delta_{g} \hat{W}_{\delta_{\varepsilon}(t), \xi}^{\text {in }}\right)\right.\right. \\
+2 G_{g}^{\prime}\left(\left(\nabla \hat{W}_{\delta_{\varepsilon}(t), \xi}^{\text {in }}\right)^{\prime}-\left(\nabla \hat{W}_{\delta_{\varepsilon}(t), \xi}^{\text {out }}\right)\right. \\
=O\left(\delta_{\varepsilon}(t)^{\frac{n}{2}}\right),
\end{gathered}
$$


and

$$
\left(\Delta W_{\delta_{\varepsilon}(t), \xi}^{\text {in }}-\Delta W_{\delta_{\varepsilon}(t), \xi}^{\text {out }}\right)=G_{g} \Delta_{g}\left(\hat{W}_{\delta_{\varepsilon}(t), \xi}^{\text {in }}-\hat{W}_{\delta_{\varepsilon}(t), \xi}^{\text {out }}\right)=O\left(\delta_{\varepsilon}(t)^{\frac{n}{2}}\right) .
$$

We observe, since $\hat{W}_{\delta_{\varepsilon}(t), \xi}=O\left(\delta_{\varepsilon}(t)^{\frac{n-4}{2}}\right)$ on $M \backslash B_{\xi}\left(r_{0}\right)$ and $P_{g} G_{g}(., \xi)=0$, that

$$
\begin{aligned}
f_{\varepsilon}\left(W_{\delta_{\varepsilon}(t), \xi}-G_{g}(., \xi) \Gamma\right)- & P_{g}\left(W_{\delta_{\varepsilon}(t), \xi}-G_{g}(., \xi) \Gamma\right) \\
& =O\left(\delta_{\varepsilon}(t)^{\frac{n+4}{2}}+\varepsilon \delta_{\varepsilon}(t)^{\frac{n-4}{2}}\right), \text { on } M \backslash B_{\xi}\left(r_{0}\right),
\end{aligned}
$$

where $\Gamma(x)=\gamma_{\tilde{\varepsilon}}\left(d(x, \xi)-r_{0}\right)\left(\hat{W}_{\delta_{\varepsilon}(t), \xi}^{i n}\right)^{\prime}\left(r_{0}\right)$. Next, using the properties of $\gamma_{\tilde{\varepsilon}}$ and the fact that $n \geq 5$, it is easy to see that

$$
\int_{M \backslash B_{\xi}\left(r_{0}\right)} P_{g}\left(G_{g}(., \xi) \Gamma\right) \phi d V=\int_{B_{\xi}\left(r_{0}+\tilde{\varepsilon}\right) \backslash B_{\xi}\left(r_{0}\right)} P_{g}\left(G_{g}(., \xi) \Gamma\right) \phi d V=O(\tilde{\varepsilon}),
$$

and

$$
\int_{M \backslash B_{\xi}\left(r_{0}\right)} f_{\varepsilon}\left(G_{g}(., \xi) \Gamma\right) \phi d V=O(\tilde{\varepsilon}) .
$$

Therefore, by Sobolev's and trace's embeddings, and choosing $\tilde{\varepsilon} \leq \delta_{\varepsilon}(t)^{\frac{n}{2}}$, we deduce that

$$
\begin{aligned}
\| i^{*}\left(f_{\varepsilon}\left(W_{\delta_{\varepsilon}(t), \xi}\right)\right)- & W_{\delta_{\varepsilon}(t), \xi} \|_{H^{2}(M)} \\
& \leq\left\|f_{\varepsilon}\left(W_{\delta_{\varepsilon}(t), \xi}\right)-P_{g} W_{\delta_{\varepsilon}(t), \xi}\right\|_{L^{\frac{2 n}{n+4}\left(B_{\xi}\left(r_{0}\right)\right)}}+O\left(\delta_{\varepsilon}(t)^{\frac{n}{2}}\right) .
\end{aligned}
$$

Since $M$ is l.c.f., using the conformal invariance of $P_{g}$ and doing some computations, we find

$$
\begin{aligned}
P_{g}\left(W_{\delta_{\varepsilon}(t), \xi}\right)= & \beta_{n} G_{g} r^{n-4} \delta_{\varepsilon}(t)^{-\frac{n+4}{2}} U\left(\delta^{-1} y\right)^{2^{*}-1} \\
+ & \frac{4 A_{\xi} \delta_{\varepsilon}(t)^{2} \beta_{n} \alpha_{n} \delta_{\varepsilon}(t)^{\frac{n-4}{2}}(n-4)^{2}|y|^{n-8}}{\left(\delta_{\varepsilon}(t)^{2}+|y|^{2}\right)^{\frac{n+2}{2}}} \\
& \times\left(\delta_{\varepsilon}(t)^{4}\left(18-9 n+n^{2}\right)-8 \delta_{\varepsilon}(t)^{2}(n-3)|y|^{2}-(n-6)|y|^{4}\right) \\
& + \text { h.o.t. }
\end{aligned}
$$

Here and in the following, h.o.t. stands for a term which is asymptotically smaller than one of the previous terms in the expansion as $\varepsilon$ goes to 0 . Therefore, we deduce that

$$
\begin{aligned}
& \left\|f_{\varepsilon}\left(W_{\delta_{\varepsilon}(t), \xi}\right)-P_{g} W_{\delta_{\varepsilon}(t), \xi}\right\|_{L^{\frac{2 n}{n+4}\left(B_{\xi}\left(r_{0}\right)\right)}} \\
& =O\left(\left\|\frac{\delta_{\varepsilon}(t)^{\frac{n}{2}}|y|^{n-8}}{\left(\delta_{\varepsilon}(t)^{2}+|y|^{2}\right)^{\frac{n+2}{2}}}\left(\delta_{\varepsilon}(t)^{4}+\delta_{\varepsilon}(t)^{2}|y|^{2}+|y|^{4}\right)\right\|_{L^{\frac{2 n}{n+4}\left(B_{\xi}\left(r_{0}\right)\right)}}\right) \\
& +\varepsilon\left\|\operatorname{div}_{g}\left(B\left(\nabla W_{\delta_{\varepsilon}(t), \xi}\right)\right)\right\|_{L^{\frac{2 n}{n+4}\left(B_{\xi}\left(r_{0}\right)\right)}} \text {. }
\end{aligned}
$$


Now, rough estimates give

$$
\begin{array}{r}
\left\|\frac{\delta_{\varepsilon}(t)^{\frac{n}{2}}|y|^{n-8}}{\left(\delta_{\varepsilon}(t)^{2}+|y|^{2}\right)^{\frac{n+2}{2}}}\left(\delta_{\varepsilon}(t)^{4}+\delta_{\varepsilon}(t)^{2}|y|^{2}+|y|^{4}\right)\right\|_{L^{\frac{2 n}{n+4}}\left(B_{\xi}\left(r_{0}\right)\right)} \\
\leq C \begin{cases}\delta_{\varepsilon}(t)^{n-4}, & \text { if } n<8, \\
\delta_{\varepsilon}(t)^{4}\left|\ln \delta_{\varepsilon}(t)\right|^{\frac{3}{4}}, & \text { if } n=8, \\
\delta_{\varepsilon}(t)^{\frac{n}{2}}, & \text { if } n>8 .\end{cases}
\end{array}
$$

and

$$
\varepsilon\left\|\operatorname{div}_{g}\left(B\left(\nabla W_{\delta_{\varepsilon}(t), \xi}\right)\right)\right\|_{L^{\frac{2 n}{n+4}}\left(B_{\xi}\left(r_{0}\right)\right)} \leq C \begin{cases}\varepsilon \delta_{\varepsilon}(t)^{\frac{n-4}{2}}, & \text { if } n<8 \\ \varepsilon \delta_{\varepsilon}(t)^{2}\left|\ln \delta_{\varepsilon}(t)\right|^{\frac{n+4}{2 n}}, & \text { if } n=8 \\ \varepsilon \delta_{\varepsilon}(t)^{2}, & \text { if } n>8\end{cases}
$$

Combining all the previous estimates, we conclude that

$$
\begin{aligned}
&\left\|f_{\varepsilon}\left(W_{\delta_{\varepsilon}(t), \xi}\right)-P_{g} W_{\delta_{\varepsilon}(t), \xi}\right\|_{L^{\frac{2 n}{n+4}}\left(B_{\xi}\left(r_{0}\right)\right)} \\
&= \begin{cases}\delta_{\varepsilon}(t)^{n-4}+\varepsilon \delta_{\varepsilon}(t)^{\frac{n-4}{2}}, & \text { if } n<8, \\
\delta_{\varepsilon}(t)^{4}\left|\ln \delta_{\varepsilon}(t)\right|^{\frac{3}{4}}+\varepsilon \delta_{\varepsilon}(t)^{2}\left|\ln \delta_{\varepsilon}(t)\right|^{\frac{3}{4}}, & \text { if } n=8, \\
\delta_{\varepsilon}(t)^{\frac{n}{2}}+\varepsilon \delta_{\varepsilon}(t)^{2}, & \text { ifn }>8 .\end{cases}
\end{aligned}
$$

Finally, we give an estimate of the reduced energy $\tilde{I}_{\varepsilon}(t, \xi)$.

Proposition 4.2. Assume that $M$ is l.c.f. and

$f_{\varepsilon}(u)=|u|^{2^{*}-2} u-\varepsilon \operatorname{div}_{g}(B(\nabla u))$. There exist constants $c_{i}(n), i=1,2,3$ depending on $n$, such that

$$
\begin{aligned}
& \tilde{I}_{\varepsilon}(t, \xi) \\
& \quad=c_{1}(n)+ \begin{cases}e^{-\frac{2 t}{\varepsilon}}\left(c_{2}(n) t \operatorname{Tr}_{g} B(\xi)-c_{3} A_{\xi}\right)+o\left(e^{-\frac{2 t}{\varepsilon}}\right), & \text { if } n=6, \\
\varepsilon^{\frac{n-4}{n-6}}\left(c_{2}(n) t^{2} \operatorname{Tr}_{g} B(\xi)-c_{3}(n) t^{n-4} A_{\xi}\right)+o\left(\varepsilon^{\frac{n-4}{n-6}}\right), & \text { if } n \geq 7\end{cases}
\end{aligned}
$$

as $\varepsilon \rightarrow 0 C^{0}$ uniformly with respect to $t$ in compact subsets of $\mathbb{R}_{+}^{*}$ and with respect to $\xi \in M$ and $C^{1}$ uniformly if $n \geq 7$. Moreover, we have that $c_{2}(n)>0$ and $c_{3}(n)>0$.

Proof. As previously, using Proposition ??, Lemma ?? and the definition of $\delta_{\varepsilon}(t)$ (given in (??)), it is easy to see that

$$
\tilde{I}_{\varepsilon}(t, \xi)=\tilde{J}_{\varepsilon}\left(W_{\delta_{\varepsilon}(t), \xi}\right)+o\left(\delta_{\varepsilon}(t)^{n-4}\right) .
$$


Using (??) and (??), we have

$$
\begin{aligned}
\int_{M} & \left(\Delta_{g} W_{\delta_{\varepsilon}(t), \xi}\right)^{2} d V+\int_{M} A_{g}\left(\nabla W_{\delta_{\varepsilon}(t), \xi}, \nabla W_{\delta_{\varepsilon}(t), \xi}\right) d V+\int_{M} h W_{\delta_{\varepsilon}(t), \xi}^{2} d V \\
= & \int_{B_{\xi}\left(r_{0}\right)} P_{g}\left(W_{\delta_{\varepsilon}(t), \xi}\right) W_{\delta_{\varepsilon}(t), \xi} d V+O\left(\delta_{\varepsilon}(t)^{n-2}\right) .
\end{aligned}
$$

Here we also used (??), (??) and $W_{\delta_{\varepsilon}(t), \xi}=O\left(\delta_{\varepsilon}(t)^{\frac{n-4}{2}}\right)$ on $\partial B_{\xi}\left(r_{0}\right)$. Now using (??), we find

$$
\begin{aligned}
P_{g}\left(W_{\delta_{\varepsilon}(t), \xi}\right) W_{\delta_{\varepsilon}(t), \xi}= & \alpha_{n}^{2^{*}} \frac{\delta_{\varepsilon}(t)^{n}}{\left(\delta_{\varepsilon}(t)^{2}+r^{2}\right)^{n}}+2 A_{\xi} \beta_{n} \alpha_{n}^{2^{*}} r^{n-4} \frac{\delta_{\varepsilon}(t)^{n}}{\left(\delta_{\varepsilon}(t)^{2}+r^{2}\right)^{n}} \\
+ & 4(n-4)^{2} A_{\xi} \beta_{n} \alpha_{n}^{2} r^{n-8} \frac{\delta_{\varepsilon}(t)^{n-2}}{\left(\delta_{\varepsilon}(t)^{2}+r^{2}\right)^{n-1}} \\
& \times\left(\left(n^{2}-9 n-18\right) \delta^{4}-8(n-3) \delta_{\varepsilon}(t)^{2} r^{2}-(n-6) r^{4}\right) \\
& + \text { h.o.t. }
\end{aligned}
$$

Integrating the previous formula, we find

$$
\begin{aligned}
& \int_{B_{\xi}\left(r_{0}\right)} P_{g}\left(W_{\delta_{\varepsilon}(t), \xi}\right) W_{\delta_{\varepsilon}(t), \xi} d V \\
&= \frac{\alpha^{2^{*}} \omega_{n-1}}{2} I_{n}^{\frac{n-2}{2}}+A_{\xi} \beta_{n} \alpha_{n}^{2^{*}} \omega_{n-1} \delta_{\varepsilon}(t)^{n-4} I_{n}^{n-3} \\
&+\left(\left(n^{2}-9 n-18\right) I_{n-1}^{n-5}-8(n-3) I_{n-1}^{n-4}-(n-6) I_{n-1}^{n-3}\right) \\
& \quad \times 4(n-4)^{2} A_{\xi} \beta_{n} \alpha_{n}^{2} \frac{\delta_{\varepsilon}(t)^{n-4} \omega_{n-1}}{2}+o\left(\delta_{\varepsilon}(t)^{n-4}\right) \\
&=\alpha^{2^{*}} \frac{\omega_{n}}{2^{n}}+\frac{A_{\xi} \beta_{n} \alpha_{n}^{2^{*}} \omega_{n-1}}{(n-1)(n-2)} \delta^{n-4} \\
& \quad+\left(\frac{2\left(n^{2}-9 n-18\right)}{(n-2)(n-3)(n-4)}-\frac{n+2}{n-2}\right) \\
& \quad \times 4(n-4)^{2} A_{\xi} \beta_{n} \alpha_{n}^{2} \frac{\delta_{\varepsilon}(t)^{n-4} \omega_{n-1}}{2}+o\left(\delta_{\varepsilon}(t)^{n-4}\right) .
\end{aligned}
$$

We also have

$$
\begin{aligned}
\int_{B_{\xi}\left(r_{0}\right)} & W_{\delta_{\varepsilon}(t), \xi}^{2^{*}} d V \\
& =\alpha_{n}^{2^{*}} \omega_{n-1} \int_{0}^{r_{0}}\left(1+\frac{2 n}{n-4} A_{\xi} \beta_{n} r^{n-4}\right) \frac{\delta_{\varepsilon}(t)^{n} r^{n-1}}{\left(\delta_{\varepsilon}(t)^{2}+r^{2}\right)^{n}} d r+o\left(\delta_{\varepsilon}(t)^{n-4}\right) \\
& =\frac{1}{2} \alpha_{n}^{2^{*}} \omega_{n-1} I_{n}^{\frac{n-2}{2}}+\frac{1}{2} \frac{2 n}{n-4} A_{\xi} \alpha_{n}^{2^{*}} \omega_{n-1} \beta_{n} \delta_{\varepsilon}(t)^{n-4} I_{n}^{n-3}+o\left(\delta_{\varepsilon}(t)^{n-4}\right)
\end{aligned}
$$


and

$$
\begin{aligned}
\varepsilon \int_{B_{\xi}\left(r_{0}\right)} & B\left(\nabla W_{\delta_{\varepsilon}(t), \xi}, \nabla W_{\delta_{\varepsilon}(t), \xi}\right) d V \\
= & \frac{(n-4)^{2}}{2 n} \varepsilon \delta_{\varepsilon}(t)^{2} \alpha_{n}^{2} \omega_{n-1} \operatorname{Tr}_{g}(B) \int_{0}^{\frac{r_{0}}{\delta_{\varepsilon}(t)}} \frac{r^{n+1}}{\left(1+r^{2}\right)^{n-2}} d r+o\left(\delta_{\varepsilon}(t)^{n-4}\right) \\
= & \frac{1}{2} \frac{(n-4)^{2}}{2 n} \varepsilon \delta_{\varepsilon}(t)^{2} \alpha_{n}^{2} \omega_{n-1} \operatorname{Tr}_{g}(B) \begin{cases}\frac{4 n(n-1)}{(n-4)(n-6)}, & \text { if } n>6, \\
\frac{1}{2} \ln \left(1+\frac{1}{\delta_{\varepsilon}(t)^{2}}\right), & \text { if } n=6\end{cases} \\
& +o\left(\delta_{\varepsilon}(t)^{n-4}\right) .
\end{aligned}
$$

Therefore, combining the three previous estimates, we finally obtain

$$
\begin{aligned}
& \tilde{J}_{\varepsilon}\left(W_{\delta_{\varepsilon}(t), \xi}\right)=\frac{4}{n} \alpha^{2^{*}} \omega_{n}+\frac{A_{\xi} \beta_{n} \alpha_{n}^{2^{*}} \omega_{n-1}}{4(n-1)(n-2)} \delta_{\varepsilon}(t)^{n-4} \\
& +2(n-4)^{2} A_{\xi} \beta_{n} \alpha_{n}^{2} \frac{\delta_{\varepsilon}(t)^{n-4} \omega_{n-1}}{2} \\
& \times\left(\frac{2\left(n^{2}-9 n-18\right)}{(n-2)(n-3)(n-4)}-\frac{(n+2)}{(n-2)}\right) \\
& +\frac{(n-4)^{2}}{8 n} \varepsilon \delta_{\varepsilon}(t)^{2} \alpha_{n}^{2} \omega_{n-1} \operatorname{Tr}_{g}(B) \begin{cases}\frac{4 n(n-1)}{(n-4)(n-6)}, & \text { if } n>6, \\
\ln \left|\delta_{\varepsilon}(t)\right|, & \text { if }=6\end{cases} \\
& +o\left(\delta_{\varepsilon}(t)^{n-4}\right) \\
& =C_{1}-C_{2} A_{\xi} \delta_{\varepsilon}(t)^{n-4}+C_{3} \operatorname{Tr}_{g} B \varepsilon \delta_{\varepsilon}(t)^{2}+o\left(\delta_{\varepsilon}(t)^{n-4}\right),
\end{aligned}
$$

where $C_{3}>0$ and $C_{2}>0$.

The rest of the proof is devoted to prove that (??) holds $C^{1}$-uniformly if $n \geq 7$. This will be an immediate consequence of the following three lemmata. To simplify notation, in the following, we denote $Z_{0}=Z_{\delta, \xi}$ and $Z_{i}=Z_{\delta, \xi, e_{i}}$, where $\left(e_{i}\right)_{i}$ is a base of $T_{\xi} M$ (see (??) and (??) for the definitions of $Z_{\delta, \xi}$ and $\left.Z_{\delta, \xi, e_{i}}\right)$.

Lemma 4.3. For any $i=1, \ldots, n$, we have

$$
\left.\frac{d}{d \eta_{i}} \tilde{I}_{\varepsilon}\left(t, \exp _{\xi} \eta\right)\right|_{\eta=0}=\frac{C_{n} \lambda_{i}}{\delta_{\varepsilon}(t)}\left\|\Delta V_{i}\right\|_{L^{2}}^{2}+o\left(\sum_{j=0}^{n} \lambda_{j}\right),
$$

and

$$
\frac{d}{d t} \tilde{I}_{\varepsilon}(t, \xi)=\frac{C_{n} \lambda_{0} \delta_{\varepsilon}(t)^{\prime}}{4 \delta_{\varepsilon}(t)}\left\|\Delta V_{0}\right\|_{L^{2}}^{2}+o\left(\sum_{j=0}^{n} \lambda_{j}\right) .
$$


where $C_{n}=\alpha_{n}(n-4)$ (see (??) for the definition of $\alpha_{n}$ and see below for the definition of the $\lambda_{i}$ 's).

Proof. We only prove (??) (the proof of (??) following along the same line). For any $i=1, \ldots, n$, using Proposition ??, we have that there exist $\lambda_{i}$, $i=0, \ldots, n$, such that

$$
\left.\frac{d}{d \eta_{i}} \tilde{I}_{\varepsilon}\left(t, \exp _{\xi} \eta\right)\right|_{\eta=0}=\sum_{j=0}^{n} \lambda_{j}\left\langle Z_{j},\left.\frac{d}{d \eta_{i}}\left(W_{\delta_{\varepsilon}(t), \exp _{\xi} \eta}+\phi_{\delta_{\varepsilon}(t), \exp _{\xi} \eta}\right)\right|_{\eta=0}\right\rangle .
$$

To simplify notation, we denote $\left.\frac{d}{d \eta_{i}}\left(W_{\delta_{\varepsilon}(t), \exp _{\xi} \eta}\right)\right|_{\eta=0}=\frac{d}{d \eta_{i}} W$ (and we adopt the same convention for all functions). Integrating by parts, we have, for all $i=1, \ldots, n$ and $j=0, \ldots n$,

$$
\left\langle Z_{j}, \frac{d}{d \eta_{i}} W\right\rangle=\int_{M} P_{g} Z_{j}\left(\frac{d}{d \eta_{i}} W-\frac{C_{n}}{\delta_{\varepsilon}(t)} Z_{i}\right) d V+\frac{C_{n}}{\delta_{\varepsilon}(t)}\left\langle Z_{i}, Z_{j}\right\rangle .
$$

Using Hölder's inequality and since $\left\|\frac{d}{d \eta_{i}} W-\frac{C_{n}}{\delta_{\varepsilon}(t)} Z_{i}\right\|_{L^{2^{*}}}=o(1)$, we get

$$
\left|\int_{M} P_{g} Z_{j}\left(\frac{d}{d \eta_{i}} W-\frac{C_{n}}{\delta_{\varepsilon}(t)} Z_{i}\right) d V\right| \leq\left\|P_{g} Z_{j}\right\|_{L^{\frac{2 n}{n+4}}}\left\|\frac{d}{d \eta_{i}} W-\frac{C_{n}}{\delta_{\varepsilon}(t)} Z_{i}\right\|_{L^{2^{*}}}=o(1) .
$$

Therefore, we deduce from the two previous lines that

$$
\left\langle Z_{j}, \frac{d}{d \eta_{i}} W\right\rangle=\frac{C_{n}}{\delta_{\varepsilon}(t)}\left\|\Delta V_{i}\right\|_{L^{2}}^{2} \delta_{i j}+o(1)
$$

On the other hand, using that $\phi_{\delta_{\varepsilon}(t), \xi} \in K_{\delta_{\varepsilon}(t), \xi}^{\perp}$, Hölder inequality and Lemma ??, we deduce that

$$
\left|\left\langle Z_{j}, \frac{d}{d \eta_{i}} \phi\right\rangle\right| \leq\left\|\frac{d}{d \eta_{i}} Z_{j}\right\|_{P_{g}}\left\|\phi_{\delta_{\varepsilon}(t), \xi}\right\|_{P_{g}}=O\left(\frac{\left\|\phi_{\delta_{\varepsilon}(t), \xi}\right\|_{P_{g}}}{\delta_{\varepsilon}(t)}\right)=o(1) .
$$

Combining (??) and (??), we get (??).

Lemma 4.4. We have

$$
\begin{aligned}
\frac{d}{d t} \tilde{J}_{\varepsilon}\left(W_{\delta_{\varepsilon}(t), \xi}\right)=C_{n} \frac{\delta_{\varepsilon}(t)^{\prime}}{4 \delta_{\varepsilon}(t)} D \tilde{J}_{\varepsilon}\left(W_{\delta_{\varepsilon}(t), \xi}\right) Z_{0} & \\
+ & + \begin{cases}o\left(\delta_{\varepsilon}(t)^{2} \ln \delta_{\varepsilon}(t)\right), & \text { if } n=6, \\
O\left(\delta_{\varepsilon}(t)^{n-2}+\varepsilon \delta_{\varepsilon}(t)^{n-4}\right), & \text { if } n>6\end{cases}
\end{aligned}
$$


and

$$
\begin{aligned}
\left.\frac{d}{d \eta_{i}} \tilde{J}_{\varepsilon}\left(W_{\delta_{\varepsilon}(t), \exp _{\xi} \eta}\right)\right|_{\eta=0}= & \frac{C_{n}}{\delta_{\varepsilon}(t)} D \tilde{J}_{\varepsilon}\left(W_{\delta_{\varepsilon}(t), \xi}\right) Z_{i} \\
& + \begin{cases}o\left(\delta_{\varepsilon}(t)^{2}\right), & \text { if } n=6, \\
O\left(\delta_{\varepsilon}(t)^{n-3}+\varepsilon \delta_{\varepsilon}(t)^{3} \ln \delta_{\varepsilon}(t)\right), & \text { if } n>6 .\end{cases}
\end{aligned}
$$

Proof. Integrating by parts, we have

$$
\begin{aligned}
\frac{d}{d t} \tilde{J}_{\varepsilon}\left(W_{\delta_{\varepsilon}(t), \xi}\right) & -C_{n} \frac{\delta_{\varepsilon}(t)^{\prime}}{4 \delta_{\varepsilon}(t)} D \tilde{J}_{\varepsilon}\left(W_{\delta_{\varepsilon}(t), \xi}\right) Z_{0} \\
& =\int_{M}\left(P_{g} W_{\delta_{\varepsilon}(t), \xi}-f_{\varepsilon}\left(W_{\delta_{\varepsilon}(t), \xi}\right)\right)\left(\frac{d}{d t} W_{\delta_{\varepsilon}(t), \xi}-C_{n} \frac{\delta_{\varepsilon}(t)^{\prime}}{4 \delta_{\varepsilon}(t)} Z_{0}\right) d V \\
& -\int_{\partial B_{\xi}\left(r_{0}\right)}\left(\Delta_{g} W_{\delta_{\varepsilon}(t), \xi}^{\text {out }} \partial_{\text {in }} \frac{d}{d t} W_{\delta_{\varepsilon}(t), \xi}+\Delta_{g} W_{\delta_{\varepsilon}(t), \xi}^{\text {in }} \partial_{\text {out }} \frac{d}{d t} W_{\delta_{\varepsilon}(t), \xi}\right) d \sigma \\
& +\int_{\partial B_{\xi}\left(r_{0}\right)}\left(\partial_{\text {in }}\left(\Delta_{g} W_{\delta_{\varepsilon}(t), \xi}^{\text {out }}\right)+\partial_{\text {out }}\left(\Delta_{g} W_{\delta_{\varepsilon}(t), \xi}^{\text {in }}\right)\right) \frac{d}{d t} W_{\delta_{\varepsilon}(t), \xi} d \sigma .
\end{aligned}
$$

Since $\frac{d}{d t} W_{\delta_{\varepsilon}(t), \xi}-C_{n} \frac{\delta_{\varepsilon}(t)^{\prime}}{4 \delta_{\varepsilon}(t)} Z_{0}=O\left(\delta_{\varepsilon}(t)^{\frac{n-6}{2}} \delta_{\varepsilon}(t)^{\prime} 1_{M \backslash B_{\xi}\left(\frac{r_{0}}{2}\right)}\right)$, using estimates obtained in Lemma ??, we find that

$$
\begin{gathered}
\int_{M}\left(P_{g} W_{\delta_{\varepsilon}(t), \xi}-f_{\varepsilon}\left(W_{\delta_{\varepsilon}(t), \xi}\right)\right)\left(\frac{d}{d t} W_{\delta_{\varepsilon}(t), \xi}-C_{n} \frac{\delta_{\varepsilon}(t)^{\prime}}{2 \delta_{\varepsilon}(t)} Z_{0}\right) d V \\
=O\left(\delta_{\varepsilon}(t)^{\prime}\left(\delta_{\varepsilon}(t)^{n-3}+\varepsilon \delta_{\varepsilon}(t)^{n-5}\right)\right), \text { if } n \geq 6 .
\end{gathered}
$$

We also have, on $\partial B_{\xi}\left(r_{0}\right)$,

$$
\Delta_{g} W_{\delta_{\varepsilon}(t), \xi}^{\text {out }} \partial_{i n} \frac{d}{d t} W_{\delta_{\varepsilon}(t), \xi}+\Delta_{g} W_{\delta_{\varepsilon}(t), \xi}^{\text {in }} \partial_{\text {out }} \frac{d}{d t} W_{\delta_{\varepsilon}(t), \xi}=O\left(\delta_{\varepsilon}(t)^{n-3} \delta_{\varepsilon}(t)^{\prime}\right)
$$

and

$$
\left(\partial_{\text {in }}\left(\Delta_{g} W_{\delta_{\varepsilon}(t), \xi}^{\text {out }}\right)+\partial_{\text {out }}\left(\Delta_{g} W_{\delta_{\varepsilon}(t), \xi}^{\text {in }}\right)\right) \frac{d}{d t} W_{\delta_{\varepsilon}(t), \xi}=O\left(\delta_{\varepsilon}(t)^{n-3} \delta_{\varepsilon}(t)^{\prime}\right) .
$$


This proves (??). Now, let us show (??). Integrating by parts, we have

$$
\begin{aligned}
\frac{d}{d \eta_{i}} \tilde{J}_{\varepsilon}(W)- & C_{n} \frac{1}{\delta_{\varepsilon}(t)} D \tilde{J}_{\varepsilon}\left(W_{\delta_{\varepsilon}(t), \xi}\right) Z_{i} \\
= & \int_{M}\left(P_{g} W_{\delta_{\varepsilon}(t), \xi}-f_{\varepsilon}\left(W_{\delta_{\varepsilon}(t), \xi}\right)\right)\left(\frac{d}{d \eta_{i}} W-C_{n} \frac{1}{\delta_{\varepsilon}(t)} Z_{i}\right) d V \\
& \quad-\int_{\partial B_{\xi}\left(r_{0}\right)}\left(\Delta_{g} W_{\delta_{\varepsilon}(t), \xi}^{\text {out }} \partial_{\text {in }} \frac{d}{d \eta_{i}} W+\Delta_{g} W_{\delta_{\varepsilon}(t), \xi}^{\text {in }} \partial_{\text {out }} \frac{d}{d \eta_{i}} W\right) d \sigma \\
& \quad \int_{\partial B_{\xi}\left(r_{0}\right)}\left(\partial_{\text {in }}\left(\Delta_{g} W_{\delta_{\varepsilon}(t), \xi}^{\text {out }}\right)+\partial_{\text {out }}\left(\Delta_{g} W_{\delta_{\varepsilon}(t), \xi}^{\text {in }}\right)\right) \frac{d}{d \eta_{i}} W d \sigma .
\end{aligned}
$$

Proceeding exactly as in Lemma 6.1 of [?], we have for any $y \in B_{0}\left(r_{0}\right)$,

$$
\left.\frac{d}{d \eta_{i}} W_{\delta_{\varepsilon}(t), \exp _{\xi} \eta}\left(\exp _{\xi} y\right)\right|_{\eta=0}=\frac{C_{n}}{\delta_{\varepsilon}(t)} Z_{i}\left(\exp _{\xi} y\right)+O\left(\frac{|y| \delta_{\varepsilon}(t)^{\frac{n-4}{2}}}{\left(\delta_{\varepsilon}(t)^{2}+|y|^{2}\right)^{\frac{n-4}{2}}}\right)
$$

For $y \in M \backslash \bar{B}_{\xi}\left(r_{0}\right)$ we have

$$
\left.\frac{d}{d \eta_{i}} W_{\delta_{\varepsilon}(t), \exp _{\xi} \eta}\left(\exp _{\xi} y\right)\right|_{\eta=0}=O\left(\delta_{\varepsilon}(t)^{\frac{n-4}{2}}\right)
$$

here we assumed to simplify computations that the function $\gamma_{\tilde{\varepsilon}} \equiv 0$, it is easy to check that this assumption is harmless since $n \geq 6$. Using (??), one can show that

$$
\Delta_{g} W_{\delta_{\varepsilon}(t), \xi}^{\text {out }} \partial_{i n} \frac{d}{d \eta_{i}} W+\Delta_{g} W_{\delta_{\varepsilon}(t), \xi}^{\text {in }} \partial_{\text {out }} \frac{d}{d \eta_{i}} W=O\left(\delta_{\varepsilon}(t)^{n-2}\right),
$$

and

$$
\left(\partial_{i n}\left(\Delta_{g} W_{\delta_{\varepsilon}(t), \xi}^{\text {out }}\right)+\partial_{\text {out }}\left(\Delta_{g} W_{\delta_{\varepsilon}(t), \xi}^{\text {in }}\right)\right) \frac{d}{d \eta_{i}} W_{\delta_{\varepsilon}(t), \xi}=O\left(\delta_{\varepsilon}(t)^{n-2}\right)
$$

Next, using (??) and (??), we obtain

$$
\begin{aligned}
& \int_{B_{\xi}\left(r_{0}\right)}\left(P_{g} W_{\delta_{\varepsilon}(t), \xi}-f_{0}\left(W_{\delta_{\varepsilon}(t), \xi}\right)\right)\left(\frac{d}{d \eta_{i}} W-C_{n} \frac{1}{\delta_{\varepsilon}(t)} Z_{i}\right) d V \\
& =O\left(\delta_{\varepsilon}(t)^{n-2} \int_{0}^{r_{0}} \frac{r^{2 n-8}}{\left(\delta_{\varepsilon}(t)^{2}+r^{2}\right)^{n-1}}\left(\delta_{\varepsilon}(t)^{4}+\delta_{\varepsilon}(t)^{2} r^{2}+r^{4}\right) d r\right) \\
& =O\left(\delta_{\varepsilon}(t)^{n-3}\right),
\end{aligned}
$$


and

$$
\begin{array}{r}
\int_{B_{\xi}\left(r_{0}\right)} B\left(\nabla W_{\delta_{\varepsilon}(t), \xi}, \nabla\left(\frac{d}{d \eta_{i}} W-C_{n} \frac{1}{\delta_{\varepsilon}(t)} Z_{i}\right) d V\right. \\
=O\left(\begin{array}{ll}
\delta_{\varepsilon}(t)^{2}, & \text { if } n=6, \\
\delta_{\varepsilon}(t)^{3} \ln \delta_{\varepsilon}(t), & \text { if } n=7, \\
\delta_{\varepsilon}(t)^{3}, & \text { if } n>7
\end{array}\right)
\end{array}
$$

Finally, using (??), we get

$$
\begin{aligned}
\int_{M \backslash B_{\xi}\left(r_{0}\right)} & \left(P_{g} W_{\delta_{\varepsilon}(t), \xi}-f_{\varepsilon}\left(W_{\delta_{\varepsilon}(t), \xi}\right)\right)\left(\frac{d}{d \eta_{i}} W-C_{n} \frac{1}{\delta_{\varepsilon}(t)} Z_{i}\right) d V \\
= & O\left(\delta_{\varepsilon}(t)^{n-2}+\varepsilon \delta_{\varepsilon}(t)^{n-4}\right) .
\end{aligned}
$$

Combining the previous estimates, we obtain (??).

Lemma 4.5. For any $i=0, \ldots, n$, we have

$$
\lambda_{i}=\frac{D \tilde{J}_{\varepsilon}\left(W_{\delta_{\varepsilon}(t), \xi}\right) \cdot Z_{i}}{\left\|\Delta V_{i}\right\|_{L^{2}}^{2}}+\left\{\begin{array}{ll}
o\left(\varepsilon \delta_{\varepsilon}(t)^{2}\right) & \text { if } i=0 \text { and } n \geq 7 \\
o\left(\varepsilon \delta_{\varepsilon}(t)^{3}\right) & \text { if } i \neq 0 \text { and } n \geq 7
\end{array} .\right.
$$

In particular, we have, for all $i=0, \ldots, n$ and $n \geq 7$,

$$
\lambda_{i}=O\left(\varepsilon \delta_{\varepsilon}(t)^{2}\right) .
$$

Proof. For any $i=0, \ldots, n$, we have

$$
D \tilde{J}_{\varepsilon}\left(W_{\delta_{\varepsilon}(t), \xi}+\phi_{\delta_{\varepsilon}(t), \xi}\right) Z_{i}=\sum_{j=0}^{n} \lambda_{j}\left\langle Z_{i}, Z_{j}\right\rangle=\lambda_{i}\left\|\nabla V_{i}\right\|_{2}^{2}+o\left(\sum_{j=0}^{n} \lambda_{i}\right)
$$

Independently, we obtain that

$$
\begin{aligned}
& D \tilde{J}_{\varepsilon}\left(W_{\delta_{\varepsilon}(t), \xi}+\phi_{\delta_{\varepsilon}(t), \xi}\right) Z_{i}=D \tilde{J}_{\varepsilon}\left(W_{\delta_{\varepsilon}(t), \xi}\right) Z_{i} \\
&+\left\langle Z_{i}-i^{*}\left(f_{\varepsilon}^{\prime}\left(W_{\delta_{\varepsilon}(t), \xi}\right) Z_{i}\right), \phi_{\delta_{\varepsilon}(t), \xi}\right\rangle \\
&-\int_{M}\left(f_{0}\left(W_{\delta_{\varepsilon}(t), \xi}+\phi\right)-f_{0}\left(W_{\delta_{\varepsilon}(t), \xi}\right)-f_{0}^{\prime}\left(W_{\delta_{\varepsilon}(t), \xi}\right) \phi_{\delta_{\varepsilon}(t), \xi}\right) Z_{i} d V
\end{aligned}
$$

Using Hölder inequality, we get

$$
\left\langle Z_{i}-i^{*}\left(f_{\varepsilon}^{\prime}\left(W_{\delta_{\varepsilon}(t), \xi}\right) Z_{i}\right), \phi_{\delta_{\varepsilon}(t), \xi}\right\rangle \leq\left\|Z_{i}-i^{*}\left(f_{\varepsilon}^{\prime}\left(W_{\delta_{\varepsilon}(t), \xi}\right) Z_{i}\right)\right\|_{P_{g}}\left\|\phi_{\delta_{\varepsilon}(t), \xi}\right\|_{P_{g}},
$$


and, as in (??),

$$
\begin{array}{r}
\int_{M}\left(f_{0}\left(W_{\delta_{\varepsilon}(t), \xi}+\phi_{\delta_{\varepsilon}(t), \xi}\right)-f_{0}\left(W_{\delta_{\varepsilon}(t), \xi}\right)-f_{0}^{\prime}\left(W_{\delta_{\varepsilon}(t), \xi}\right) \phi_{\delta_{\varepsilon}(t), \xi}\right) Z_{i} d V \\
\leq O\left(\left\|\phi_{\delta_{\varepsilon}(t), \xi}\right\|_{L^{\frac{2 n}{n-4}}}^{2}\right) \text { when } 6 \leq n .
\end{array}
$$

To conclude, we only have to estimate $\left\|Z_{i}-i^{*}\left(f_{\varepsilon}^{\prime}\left(W_{\delta_{\varepsilon}(t), \xi}\right) Z_{i}\right)\right\|_{P_{g}}$. First, by Sobolev's embedding, we have

$$
\left\|Z_{i}-i^{*}\left(f_{\varepsilon}^{\prime}\left(W_{\delta_{\varepsilon}(t), \xi}\right) Z_{i}\right)\right\|_{P_{g}} \leq C\left\|P_{g} Z_{i}-f_{\varepsilon}^{\prime}\left(W_{\delta_{\varepsilon}(t), \xi}\right) Z_{i}\right\|_{L^{\frac{2 n}{n+4}}} .
$$

Straight forward computations using that $M$ is l.c.f. and $P_{g}$ is conformally invariant give

$$
\begin{aligned}
& P_{g} Z_{0}-f_{0}^{\prime}\left(W_{\delta_{\varepsilon}(t), \xi}\right) Z_{0}=O\left(\delta_{\varepsilon}(t)^{\frac{n-4}{2}}\right. \\
& \left.\quad+\frac{|y|^{n-8} \delta_{\varepsilon}(t)^{\frac{n-4}{2}}}{\left(|y|^{2}+\delta_{\varepsilon}(t)^{2}\right)^{\frac{n+4}{2}}}\left(\delta_{\varepsilon}(t)^{8}+\delta_{\varepsilon}(t)^{6}|y|^{2}+\delta_{\varepsilon}(t)^{4}|y|^{4}+\delta_{\varepsilon}(t)^{2}|y|^{6}+|y|^{8}\right)\right),
\end{aligned}
$$

and, for $i=1, \ldots, n$,

$$
\begin{aligned}
P_{g} Z_{i}-f_{0}^{\prime}\left(W_{\delta_{\varepsilon}(t), \xi}\right) Z_{i}=O\left(\delta_{\varepsilon}(t)^{\frac{n-2}{2}}\right. & \\
& \left.+\frac{|y|^{n-8} \delta_{\varepsilon}(t)^{\frac{n-2}{2}}}{\left(|y|^{2}+\delta_{\varepsilon}(t)^{2}\right)^{\frac{n+4}{2}}}\left(\delta_{\varepsilon}(t)^{6}+\delta_{\varepsilon}(t)^{4}|y|^{2}+\delta_{\varepsilon}(t)^{2}|y|^{4}+|y|^{6}\right)\right) .
\end{aligned}
$$

Using the two previous estimates and that

$$
\varepsilon \| \operatorname{div}\left(B\left(\nabla Z_{i}\right) \|_{L^{\frac{2 n}{n+4}}}= \begin{cases}\varepsilon \delta_{\varepsilon}(t)^{2}\left(\ln \delta_{\varepsilon}(t)\right)^{\frac{5}{6}} & \text { if } i \neq 0, n=6, \\ \varepsilon \delta_{\varepsilon}(t)^{2}, & \text { if } i \neq 0, n>6, \\ \varepsilon \delta_{\varepsilon}(t)^{2}, & \text { if } i=0, n>8, \\ \varepsilon \delta_{\varepsilon}(t)^{2}\left(\ln \delta_{\varepsilon}(t)\right)^{\frac{3}{4}}, & \text { if } i=0, n=8, \\ \varepsilon \delta_{\varepsilon}(t)^{\frac{n-4}{2}}, & \text { if } i=0, n<8\end{cases}\right.
$$

one deduces that

$$
\left\|Z_{i}-i^{*}\left(f_{\varepsilon}^{\prime}\left(W_{\delta_{\varepsilon}(t), \xi}\right) Z_{i}\right)\right\|_{P_{g}} \leq \begin{cases}\delta_{\varepsilon}(t)^{\frac{5}{2}}+\varepsilon \delta_{\varepsilon}(t)^{\frac{5}{2}} & \text { if } i=0, n=7, \\ \delta_{\varepsilon}(t)^{2}+\varepsilon \delta_{\varepsilon}(t)^{2}\left(\ln \delta_{\varepsilon}(t)\right)^{\frac{3}{4}} & \text { if } i=0, n=8 \\ \delta_{\varepsilon}(t)^{\frac{n-4}{2}}+\varepsilon \delta_{\varepsilon}(t)^{2} & \text { if } i=0, n>8 \\ \delta_{\varepsilon}(t)^{\frac{n-2}{2}}+\varepsilon \delta_{\varepsilon}(t)^{2}, & \text { if } i \neq 0, n \geq 7 .\end{cases}
$$


Combining (??), (??), (??), (??) and using Lemma ??, we obtain (??). Finally (??) follows from the fact that the estimates we obtain for $\tilde{J}_{\varepsilon}\left(W_{\delta_{\varepsilon}(t), \xi}\right)$ in Lemma ??, are $C^{1}$-uniform with respect to $t$ in compact sets of $\mathbb{R}_{+}^{*}$ and $\xi \in M$. 